\title{
Robust Output Feedback Disturbance Rejection Control by Simultaneously Estimating State and Disturbance
}

\author{
Jeang-Lin Chang \\ Department of Electrical Engineering, Oriental Institute of Technology, Panchiao, New Taipei City 220, Taiwan \\ Correspondence should be addressed to Jeang-Lin Chang, jlchang@ee.oit.edu.tw
}

Received 21 April 2011; Accepted 31 July 2011

Academic Editor: Weizhou Su

Copyright () 2011 Jeang-Lin Chang. This is an open access article distributed under the Creative Commons Attribution License, which permits unrestricted use, distribution, and reproduction in any medium, provided the original work is properly cited.

This paper tackles the problem of simultaneous estimation of the state and the unknown disturbance of an MIMO disturbed system and designs the disturbance rejection controller according to the estimation information. Through a series of transformations, we can transform the original system into two subsystems and then propose a sliding mode observer and a descriptor system form observer, respectively. Our algorithm can simultaneously estimate the state and the unknown disturbance. The estimation error is shown to be bounded within a small region. Moreover, the controller algorithm developed in this paper can effectively avoid the peaking phenomenon. Finally, the feasibility and the performance using the proposed method are analyzed and demonstrated with two simulated examples.

\section{Introduction}

Disturbances coming from the environment often constitute an annoyance in the operation of dynamic systems. Disturbance rejection control (DRC), in which the controller is designed to suppress the disturbance's effect, is the major concern in the design of feedback control systems. Since external disturbances are usually not accessible for measurement, in the early development of disturbance rejection control, high gain control is used to suppress the unknown disturbance. Examples of high-gain controllers include the geometric control algorithm [1], the singular perturbation control laws [2], and the sliding mode control schemes [3]. When the system is minimum phase and has relative degree one, Lin and Saberi [4] have shown that the system can be semiglobally stabilizable via linear static high-gain output feedback. However, high-gain control has drawbacks such as easy control saturation as well as peaking of both state and control. Moreover, the peaking phenomenon can easily excite the unmodelled high-order dynamics [5]. Hence, the implementations of high-gain DRC in real-world applications are usually constrained.

There is another DRC method in which an observer algorithm is first proposed to estimate the unknown disturbance and then cancel the disturbance's effect by the control input. The advantage of this approach is that the disturbance canceling control does not need to be high gain. There are various formulations related to the unknown disturbance estimation. According to the transfer function approach, the disturbance observer is known to be very effective in compensating disturbances [6-8] and is very popular for robust motion control [9-11]. However, the transfer function approach cannot be used in MIMO cases, while the state space approach can be employed. Another approach for disturbance estimation involves designing a joint state and disturbance observer, where observers are constructed to simultaneously estimate both the state and the unknown disturbance. This approach was first developed in the study of unknown input observer [12-14]. In previous research [15-19], sliding mode methods have been developed from the concepts of sliding surface design and equivalent control. The so-called sliding mode observer (SMO) has been applied to the observer problem for robust estimation of the system state. In order to obtain perfect estimation, two necessary and sufficient conditions are required for the existence of a stable observer in the abovementioned approaches [12-16]. The first is that the system must be minimum phase (with respect to the relation between the output and unknown input), that is, the invariant zeros of the system must be 
located in the left-half plane. The second is a rank condition in which the relative degree of the transfer function matrix from the unknown input to the system output is one. These two important conditions limit the practical applications of the abovementioned approaches. Moreover, these previous studies did not consider the issue of measurement noise.

For the linear system having both unknown disturbance and measurement noise, we develop an observer design method, which can be successfully implemented in systems with unstable invariant zeros. With reference to $[15,20-23]$, our observer design combines SMO with the descriptor system approach. After introducing a coordinate transformation to the original system, a normal SMO and a descriptor form system observer which is the type of proportional derivative (PD) observer are first designed, respectively. When the sliding motion is guaranteed, the auxiliary information can be obtained by passing the switching term in SMO through the continuous approximation. Although the system has both unknown input and sensor noise, we can simultaneously estimate the state and the unknown disturbance using the proposed algorithm. The derivative and proportional gains are employed to attenuate the effect of disturbance and to ensure robust stability of the estimation error dynamics, respectively. As a result, the estimation error is shown to be bounded in a small region. Based on the estimations of both state and disturbance, we finally design the disturbance rejection controller. Unlike the high-gain controller, the response of control input in our disturbance rejection controller does not have the drawback of peaking at the transient time. Moreover, the proposed observer algorithm does not require the derivative of the output.

In the next section, a class of linear systems having both unknown disturbances and sensor noises is first introduced with three assumptions in relation to the system matrices. Section 3 describes a series of system transformations which can transform the original system into two subsystems. Section 4 develops the observer design for estimating simultaneously the system state and the unknown disturbance when the system is minimum phase. Moreover, the disturbance rejection controller is also presented in this section. The same controller design problem is examined in Section 5 but the system has unstable invariant zeros. To verify the developed disturbance rejection controller, two numerical examples are demonstrated in Section 6. Finally, Section 7 gives concluding remarks.

\section{Problem Formulation}

Consider an MIMO system with both unknown disturbance and measurement noise

$$
\begin{gathered}
\dot{\mathbf{x}}(t)=\overline{\mathbf{A}} \mathbf{x}(t)+\overline{\mathbf{B}} \mathbf{u}(t)+\overline{\mathbf{D}} \mathbf{d}_{e}(t), \\
\mathbf{y}(t)=\overline{\mathbf{C}} \mathbf{x}(t)+\overline{\mathbf{F}} \mathbf{n}(t),
\end{gathered}
$$

where $\mathbf{x} \in \mathbb{R}^{n}, \mathbf{u} \in \mathbb{R}^{m}, \mathbf{d}_{e} \in \mathbb{R}^{l}, \mathbf{n} \in \mathbb{R}^{k}$, and $\mathbf{y} \in$ $\mathbb{R}^{p}$ are the system state vector, the control input vector, the unknown disturbance vector, the measurement noise vector and the system output vector, respectively. Note that since matrices $\overline{\mathbf{B}}$ and $\overline{\mathbf{D}}$ may have different dimensions, the feedback control cannot completely eliminate the effect of disturbance on the system. It is more reasonable to estimate the disturbance on the control channel than to estimate the disturbance itself because we can use the control input to improve the disturbance rejection performance. To emphasize the advantage of DRC, we are concerned with the input disturbance where the disturbance comes into the system through the same channel as the control input (the so-called matching condition [3]). Hence, the plant can be rewritten as

$$
\begin{gathered}
\dot{\mathbf{x}}(t)=\overline{\mathbf{A}} \mathbf{x}(t)+\overline{\mathbf{B}}(\mathbf{u}(t)+\mathbf{d}(t)), \\
\mathbf{y}(t)=\overline{\mathbf{C}} \mathbf{x}(t)+\overline{\mathbf{F}} \mathbf{n}(t),
\end{gathered}
$$

where $\mathbf{d} \in \mathbb{R}^{m}$ is the unknown disturbance vector. Without loss of generality, we assume that $\operatorname{rank}(\overline{\mathbf{B}})=m$ and $\operatorname{rank}(\overline{\mathbf{F}})=k$ where $p \geq k+m$ and $n \geq p$. The objectives of this paper are to propose an observer method that can simultaneously estimate the system state and the unknown disturbance and to design the disturbance rejection controller according to the estimation obtained. The observer is an auxiliary dynamic system which can effectively estimate the system state at any instant in time. Unfortunately, the unknown disturbance brings the standard Luenberger observer additional difficulty in precise estimation. Busawon and Kabore [24] have further shown that the conventional Luenberger observer is not adequate for handling measurement noise. Several authors [12-14] have proposed the so-called unknown input observer design methods for estimating accurately the system state. According to the well-established sliding mode control technique, sliding mode observer methods [15-19] have been developed to robustly estimate the state. In the abovementioned design methods, the necessary and sufficient conditions for an asymptotically stable observer are that the transfer function matrix between the unknown disturbance and the system output is minimum phase and has relative degree one. The two conditions seriously limit the applicability of the abovementioned approaches. Since the two conditions are necessary and sufficient, if one of two conditions mentioned above is not satisfied, the abovementioned observer method cannot guarantee asymptotical stability. In this paper, we combine the sliding mode observer with the descriptor system transformation for simultaneous estimation of both system state and disturbance. The control law is then designed to cancel the unknown disturbance according to the disturbance estimation. With this new mechanism, the proposed estimation algorithm can be applied to nonminimum phase systems and the controller can effectively avoid the peaking phenomenon at the transient time. Before introducing the main results, the following three assumptions of system (2) are made throughout this paper.

Assumption 1. The pair $(\overline{\mathbf{C}}, \overline{\mathbf{A}})$ is detectable and the pair $(\overline{\mathbf{A}}, \overline{\mathbf{B}})$ is stabilizable. 
Assumption 2. System matrices $\overline{\mathbf{B}}$ and $\overline{\mathbf{C}}$ in (2) satisfy the following condition:

$$
\operatorname{rank}(\overline{\mathbf{C B}})=\operatorname{rank}(\overline{\mathbf{B}})=m
$$

This assumption implies that the transfer function matrix between the output and the input has relative degree one.

Assumption 3. The unknown disturbance and the measurement noise have the upper bound.

\section{System Transformation}

For linear systems with unknown inputs in the state and output equations, previous studies $[15,25]$ have developed different design methods under some important conditions of system matrices. Sharma and Aldeen [25] decoupled the unknown inputs from the rest of the system through a series of transformations. Following their work, we develop transformations to decouple the original system for designing the disturbance rejection controller. Since $\operatorname{rank}(\overline{\mathbf{F}})=k$, without loss of generality we assume that the matrix $\overline{\mathbf{F}}$ is decomposed as

$$
\overline{\mathbf{F}}=\left[\begin{array}{c}
\mathbf{0} \\
\overline{\mathbf{F}}_{2}
\end{array}\right],
$$

where $\overline{\mathbf{F}}_{2} \in \mathbb{R}^{k \times k}$ is invertible. It follows from $\operatorname{rank}(\overline{\mathbf{B}})=m$ that there exists a transformation $\mathbf{T}_{1} \in \mathbb{R}^{n \times n}$ such that the matrix $\overline{\mathbf{B}}$ can be partitioned as

$$
\mathbf{T}_{1} \overline{\mathbf{B}}=\left[\begin{array}{c}
\mathbf{B}_{1} \\
\mathbf{0}
\end{array}\right],
$$

where $\mathbf{B}_{1} \in \mathbb{R}^{m \times m}$ is invertible. Let $\left[\overline{\mathbf{C}}_{1} \overline{\mathbf{C}}_{2}\right]=\overline{\mathbf{C}}_{1}^{-1}$ where $\overline{\mathbf{C}}_{1} \in \mathbb{R}^{p \times m}$ and $\overline{\mathbf{C}}_{2} \in \mathbb{R}^{p \times(n-m)}$, and then we obtain

$$
\begin{aligned}
\operatorname{rank}(\overline{\mathbf{C B}}) & =\operatorname{rank}\left(\overline{\mathbf{C}} \mathbf{T}_{1}^{-1} \mathbf{T}_{1} \overline{\mathbf{B}}\right) \\
& =\operatorname{rank}\left(\left[\begin{array}{ll}
\overline{\mathbf{C}}_{1} & \overline{\mathbf{C}}_{2}
\end{array}\right]\left[\begin{array}{c}
\mathbf{B}_{1} \\
\mathbf{0}
\end{array}\right]\right)=\operatorname{rank}\left(\overline{\mathbf{C}}_{1} \mathbf{B}_{1}\right)=m .
\end{aligned}
$$

Since $\mathbf{B}_{1}$ is nonsingular, we can obtain $\operatorname{rank}\left(\overline{\mathbf{C}}_{1}\right)=m$ from (6). As a result, the matrix $\overline{\mathbf{C}}_{1}$ can be partitioned as follows:

$$
\overline{\mathbf{C}}_{1}=\left[\begin{array}{l}
\mathbf{C}_{1} \\
\mathbf{C}_{21}
\end{array}\right] \text {, }
$$

where $\mathbf{C}_{1} \in \mathbb{R}^{m \times m}$ is invertible and $\mathbf{C}_{21} \in \mathbb{R}^{(p-m) \times m}$. Now we define the transformation

$$
\mathbf{S}=\left[\begin{array}{cc}
\mathbf{I}_{m} & \mathbf{0} \\
-\mathbf{C}_{21} \mathbf{C}_{1}^{-1} & \mathbf{I}_{p-m}
\end{array}\right]
$$

which yields $\left[\begin{array}{cc}\mathbf{I}_{m} & 0 \\ -\mathbf{C}_{21} \mathbf{C}_{1}^{-1} & \mathbf{I}_{p-m}\end{array}\right]\left[\begin{array}{ll}\overline{\mathbf{C}}_{1} & \overline{\mathbf{C}}_{2}\end{array}\right]=\left[\begin{array}{cc}\mathbf{C}_{1} & \mathbf{C}_{12} \\ 0 & \mathbf{C}_{2}\end{array}\right]$ where $\mathbf{C}_{12} \in$ $\mathbb{R}^{m \times(n-m)}$ and $\mathbf{C}_{2} \in \mathbb{R}^{(p-m) \times(n-m)}$. Finally we introduce a transformation $\mathbf{T}_{2}=\left[\begin{array}{cc}\mathbf{I}_{m} & -\mathbf{C}_{1}^{-1} \mathbf{C}_{12} \\ 0 & \mathbf{I}_{n-m}\end{array}\right] \in \mathbb{R}^{n \times n}$ and define

$$
\mathbf{z}=\left[\begin{array}{l}
\mathbf{z}_{1} \\
\mathbf{z}_{2}
\end{array}\right]=\mathbf{T}_{2}^{-1} \mathbf{T}_{1} \mathbf{x}, \quad\left[\begin{array}{l}
\mathbf{y}_{1} \\
\mathbf{y}_{2}
\end{array}\right]=\mathbf{S y}=\mathbf{S} \overline{\mathbf{C}} \mathbf{x}=\mathbf{S} \overline{\mathbf{C}} \mathbf{T}_{1}^{-1} \mathbf{T}_{2} \mathbf{z}
$$

where $\mathbf{z}_{1} \in \mathbb{R}^{m}, \mathbf{z}_{2} \in \mathbb{R}^{n-m}, \mathbf{y}_{1} \in \mathbb{R}^{m}$, and $\mathbf{y}_{2} \in \mathbb{R}^{p-m}$. Note that two vectors $\mathbf{y}_{1}$ and $\mathbf{y}_{2}$ are measurable. According to the abovementioned transformation (9), we define

$$
\begin{aligned}
& {\left[\begin{array}{ll}
\mathbf{A}_{11} & \mathbf{A}_{12} \\
\mathbf{A}_{21} & \mathbf{A}_{22}
\end{array}\right]=\mathbf{T}_{2}{ }^{-1} \mathbf{T}_{1} \overline{\mathbf{A}} \mathbf{T}_{1}^{-1} \mathbf{T}_{2}, \quad\left[\begin{array}{c}
\mathbf{B}_{1} \\
\mathbf{0}
\end{array}\right]=\mathbf{T}_{2}{ }^{-1} \mathbf{T}_{1} \overline{\mathbf{B}},} \\
& {\left[\begin{array}{cc}
\mathbf{C}_{1} & \mathbf{0} \\
\mathbf{0} & \mathbf{C}_{2}
\end{array}\right]=\mathbf{S} \overline{\mathbf{C}} \mathbf{T}_{1}^{-1} \mathbf{T}_{2}, \quad\left[\begin{array}{c}
\mathbf{0} \\
\mathbf{F}_{2}
\end{array}\right]=\mathrm{S} \overline{\mathbf{F}},}
\end{aligned}
$$

where $\mathbf{F}_{2} \in \mathbb{R}^{(p-m) \times k}$ is of full rank. As a result, system (2) can be transformed into the following form:

$$
\begin{aligned}
\dot{\mathbf{z}}_{1}(t) & =\mathbf{A}_{11} \mathbf{z}_{1}(t)+\mathbf{A}_{12} \mathbf{z}_{2}(t)+\mathbf{B}_{1}(\mathbf{u}(t)+\mathbf{d}(t)) \\
\dot{\mathbf{z}}_{2}(t) & =\mathbf{A}_{21} \mathbf{z}_{1}(t)+\mathbf{A}_{22} \mathbf{z}_{2}(t), \\
\mathbf{y}_{1}(t) & =\mathbf{C}_{1} \mathbf{z}_{1}(t) \\
\mathbf{y}_{2}(t) & =\mathbf{C}_{2} \mathbf{z}_{2}(t)+\mathbf{F}_{2} \mathbf{n}(t)
\end{aligned}
$$

where $\mathbf{A}_{11} \in \mathbb{R}^{m \times m}, \mathbf{A}_{12} \in \mathbb{R}^{m \times(n-m)}, \mathbf{A}_{21} \in \mathbb{R}^{(n-m) \times m}$, and $\mathbf{A}_{22} \in \mathbb{R}^{(n-m) \times(n-m)}$. This completes the process of system transformation. To apply DRC, we first propose an observer structure that utilizes the information of input $\mathbf{u}$ and output $\mathbf{y}$ to simultaneously estimate the system state $\mathbf{x}$ and the unknown disturbance $\mathbf{d}$ in (2). According to the estimation obtained, we then design the disturbance rejection controller, which can effectively avoid the peaking phenomenon in the transient time.

\section{Disturbance Rejection Controller Design for Minimum Phase Systems}

When system (2) is minimum phase and $\operatorname{rank}(\overline{\mathbf{C B}})=$ $\operatorname{rank}(\overline{\mathbf{B}})=m$, we are in the position to design the sliding mode observer and the descriptor system form observer, which can simultaneously estimate the system state $\mathbf{x}$ and the unknown disturbance d. Although the system has measurement noise, the proposed algorithm can guarantee the estimation error to be bounded within a small region. From the third equation in (11), we know $\mathbf{z}_{1}=\mathbf{C}_{1}^{-1} \mathbf{y}_{1}$ and substitute the term into the dynamic equation of $\mathbf{z}_{2}$ to obtain

$$
\begin{gathered}
\dot{\mathbf{z}}_{2}(t)=\mathbf{A}_{22} \mathbf{z}_{2}(t)+\mathbf{A}_{21} \mathbf{C}_{1}^{-1} \mathbf{y}_{1}(t), \\
\mathbf{y}_{2}(t)=\mathbf{C}_{2} \mathbf{z}_{2}(t)+\mathbf{F}_{2} \mathbf{n}(t) .
\end{gathered}
$$


System (12) can be transformed into a descriptor system form

$$
\begin{aligned}
{\left[\begin{array}{cc}
\mathbf{I}_{n-m} & \mathbf{0} \\
\mathbf{0} & \mathbf{0}
\end{array}\right]\left[\begin{array}{c}
\dot{\mathbf{z}}_{2}(t) \\
\dot{\mathbf{n}}(t)
\end{array}\right]=} & {\left[\begin{array}{cc}
\mathbf{A}_{22} & \mathbf{0} \\
\mathbf{0} & -\mathbf{I}_{k}
\end{array}\right]\left[\begin{array}{c}
\mathbf{z}_{2}(t) \\
\mathbf{n}(t)
\end{array}\right] } \\
& +\left[\begin{array}{c}
\mathbf{A}_{21} \mathbf{C}_{1}^{-1} \\
\mathbf{0}
\end{array}\right] \mathbf{y}_{1}(t)+\left[\begin{array}{c}
\mathbf{0} \\
\mathbf{I}_{k}
\end{array}\right] \mathbf{n}(t) \\
\mathbf{y}_{2}(t)= & {\left[\begin{array}{ll}
\mathbf{C}_{2} & \mathbf{F}_{2}
\end{array}\right]\left[\begin{array}{c}
\mathbf{z}_{2}(t) \\
\mathbf{n}(t)
\end{array}\right] . }
\end{aligned}
$$

Augment a vector $\mathbf{w}_{1}$ as $\mathbf{w}_{1}=\left[\begin{array}{c}\mathbf{z}_{2} \\ \mathbf{n}\end{array}\right] \in \mathbb{R}^{n-m+k}$ and define

$$
\begin{gathered}
\mathbf{E}_{1}=\left[\begin{array}{cc}
\mathbf{I}_{n-m} & \mathbf{0} \\
\mathbf{0} & \mathbf{0}
\end{array}\right] \in \mathbb{R}^{(n-m+k) \times(n-m+k),} \\
\mathbf{H}_{1}=\left[\begin{array}{cc}
\mathbf{A}_{22} & \mathbf{0} \\
\mathbf{0} & -\mathbf{I}_{k}
\end{array}\right] \in \mathbb{R}^{(n-m+k) \times(n-m+k)}, \\
\mathbf{N}_{1}=\left[\begin{array}{c}
\mathbf{A}_{21} \mathbf{C}_{1}^{-1} \\
\mathbf{0}
\end{array}\right] \in \mathbb{R}^{(n-m+k) \times m}, \\
\mathbf{M}_{1}=\left[\begin{array}{c}
\mathbf{0} \\
\mathbf{I}_{k}
\end{array}\right] \in \mathbb{R}^{(n-m+k) \times k}, \\
\mathbf{C}_{n}=\left[\begin{array}{ll}
\mathbf{C}_{2} & \mathbf{F}_{2}
\end{array}\right] \in \mathbb{R}^{(p-m) \times(n-m+k)} .
\end{gathered}
$$

Then we can rewrite system (13) as

$$
\begin{aligned}
& \mathbf{E}_{1} \dot{\mathbf{w}}_{1}(t)= \mathbf{H}_{1} \mathbf{w}_{1}(t)+\mathbf{N}_{1} \mathbf{y}_{1}(t)+\mathbf{M}_{1} \mathbf{n}(t) \\
& \mathbf{y}_{2}(t)=\mathbf{C}_{n} \mathbf{w}_{1}(t) .
\end{aligned}
$$

In the following, two observer algorithms including the descriptor system observer and the sliding mode observer are developed to simultaneously estimate the system state $\mathbf{x}$ and the unknown disturbance d. Boutayeb et al. [20] used the descriptor system approach to design a nonlinear observer design for simultaneously estimating the system state and the unknown input. Using the same method, Fernando and Trinh [21] designed a reduced-order functional observer of the linear systems with the disturbed input and output. Gao and Wang [22] developed an observer which can reduce the effect of measurement noises by descriptor system transformation. Their proposed method involved constructing a modified PD observer, which can asymptotically estimate the system state and the output noise at the same time. Before designing the observer for system (15), the following lemma demonstrates its detectability.
Lemma 1. If system (2) is minimum phase and $\operatorname{rank}(\overline{\mathbf{C B}})=$ $\operatorname{rank}(\overline{\mathbf{B}})=m$, then the descriptor system (15) is completely detectable, that is,

$$
\begin{aligned}
& \operatorname{rank}\left(\left[\begin{array}{c}
s \mathbf{E}_{1}-\mathbf{H}_{1} \\
\mathbf{C}_{n}
\end{array}\right]\right)=n-m+k, \quad \forall s \in \mathbb{C}^{+}, \\
& \operatorname{rank}\left(\left[\begin{array}{c}
\mathbf{E}_{1} \\
\mathbf{C}_{n}
\end{array}\right]\right)=n-m+k .
\end{aligned}
$$

System (15) is completely detectable which means that it has neither unstable finite nor infinite output decoupling zeros [22, 23].

Proof. Since system (2) is minimum phase, it follows that

$$
\operatorname{rank}\left(\left[\begin{array}{cc}
s \mathbf{I}_{n}-\overline{\mathbf{A}} & -\overline{\mathbf{B}} \\
\overline{\mathbf{C}} & \mathbf{0}
\end{array}\right]\right)=n+m, \quad \forall s \in \mathbb{C}^{+}
$$

Using the transformations described in Section 3, we can obtain

$$
\begin{aligned}
& \operatorname{rank}\left(\left[\begin{array}{ccc}
s \mathbf{I}_{n}-\overline{\mathbf{A}} & -\overline{\mathbf{B}} \\
\overline{\mathbf{C}} & \mathbf{0}
\end{array}\right]\right) \\
& =\operatorname{rank}\left(\left[\begin{array}{ccc}
s \mathbf{I}_{m}-\mathbf{A}_{11} & -\mathbf{A}_{12} & -\mathbf{B}_{1} \\
-\mathbf{A}_{21} & s \mathbf{I}_{n-m}-\mathbf{A}_{22} & \mathbf{0} \\
\mathbf{C}_{1} & \mathbf{0} & \mathbf{0} \\
\mathbf{0} & \mathbf{C}_{2} & \mathbf{0}
\end{array}\right]\right) \\
& =n+m, \quad \forall s \in \mathbb{C}^{+} .
\end{aligned}
$$

Since matrices $\mathbf{B}_{1}$ and $\mathbf{C}_{1}$ are invertible, the above equation becomes

$$
\begin{aligned}
\operatorname{rank}\left(\left[\begin{array}{cc}
s \mathbf{I}_{n}-\overline{\mathbf{A}} & -\overline{\mathbf{B}} \\
\overline{\mathbf{C}} & \mathbf{0}
\end{array}\right]\right) & =\operatorname{rank}\left(\left[\begin{array}{c}
s \mathbf{I}_{n-m}-\mathbf{A}_{22} \\
\mathbf{C}_{2}
\end{array}\right]\right)+2 m \\
& =n+m, \quad \forall s \in \mathbb{C}^{+} .
\end{aligned}
$$


Consequently, we can obtain $\operatorname{rank}\left(\left[\begin{array}{c}s \mathbf{I}_{n-m}-\mathbf{A}_{22} \\ \mathbf{C}_{2}\end{array}\right]\right)=n-m$, for all $s \in \mathbb{C}^{+}$. From $\operatorname{rank}\left(\mathbf{F}_{2}\right)=k$, it follows that

$$
\begin{aligned}
& \operatorname{rank}\left(\left[\begin{array}{c}
s \mathbf{E}_{1}-\mathbf{H}_{1} \\
\mathbf{C}_{n}
\end{array}\right]\right)=\operatorname{rank}\left(\left[\begin{array}{cc}
s \mathbf{I}_{n-m}-\mathbf{A}_{22} & \mathbf{0} \\
\mathbf{0} & \mathbf{I}_{k} \\
\mathbf{C}_{2} & \mathbf{F}_{2}
\end{array}\right]\right) \\
& =\operatorname{rank}\left(\left[\begin{array}{c}
s \mathbf{I}_{n-m}-\mathbf{A}_{22} \\
\mathbf{C}_{2}
\end{array}\right]\right)+k \\
& =n-m+k, \quad \forall s \in \mathbb{C}^{+} \text {, } \\
& \operatorname{rank}\left(\left[\begin{array}{l}
\mathbf{E}_{1} \\
\mathbf{C}_{n}
\end{array}\right]\right)=\operatorname{rank}\left(\left[\begin{array}{cc}
\mathbf{I}_{n-m} & \mathbf{0} \\
\mathbf{0} & \mathbf{0} \\
\mathbf{C}_{2} & \mathbf{F}_{2}
\end{array}\right]\right) \\
& =n-m+\operatorname{rank}\left(\mathbf{F}_{2}\right) \\
& =n-m+k \text {. }
\end{aligned}
$$

Hence, system (15) is completely detectable. The proof of the lemma is complete.

Following the above procedures, we know that system (11) can be transformed into two subsystems as

$$
\begin{gathered}
\dot{\mathbf{z}}_{1}(t)=\mathbf{A}_{11} \mathbf{z}_{1}(t)+\mathbf{A}_{12} \mathbf{z}_{2}(t)+\mathbf{B}_{1}(\mathbf{u}(t)+\mathbf{d}(t)), \\
\mathbf{y}_{1}(t)=\mathbf{C}_{1} \mathbf{z}_{1}(t), \\
\mathbf{E}_{1} \mathbf{w}_{1}(t)=\mathbf{H}_{1} \mathbf{w}_{1}(t)+\mathbf{N}_{1} \mathbf{y}_{1}(t)+\mathbf{M}_{1} \mathbf{n}(t), \\
\mathbf{y}_{2}(t)=\mathbf{C}_{n} \mathbf{w}_{1}(t) .
\end{gathered}
$$

For subsystem (22), we design a PD observer as follows:

$$
\begin{aligned}
\left(\mathbf{E}_{1}+\mathbf{K}_{1} \mathbf{C}_{n}\right) \dot{\eta}(t)= & \left(\mathbf{H}_{1}+\mathbf{L}_{1} \mathbf{C}_{n}\right) \eta(t) \\
& +\left(\left(\mathbf{H}_{1}+\mathbf{L}_{1} \mathbf{C}_{n}\right)\left(\mathbf{E}_{1}+\mathbf{K}_{1} \mathbf{C}_{n}\right)^{-1} \mathbf{K}_{1}-\mathbf{L}_{1}\right) \\
& \times \mathbf{y}_{2}(t)+\mathbf{N}_{1} \mathbf{y}_{1}(t) \\
\hat{\mathbf{w}}_{1}(t)= & \eta(t)+\left(\mathbf{E}_{1}+\mathbf{K}_{1} \mathbf{C}_{n}\right)^{-1} \mathbf{K}_{1} \mathbf{y}_{2}(t),
\end{aligned}
$$

where the gain matrices $\mathbf{K}_{1} \in \mathbb{R}^{(n-m+k) \times(p-m)}$ and $\mathbf{L}_{1} \in$ $\mathbb{R}^{(n-m+k) \times(p-m)}$ are designed in the latter, $\eta \in \mathbb{R}^{n-m+k}$ is the state of the observer and $\widehat{\mathbf{w}}_{1}=\left[\begin{array}{c}\hat{\mathbf{z}}_{2} \\ \hat{\mathbf{n}}\end{array}\right] \in \mathbb{R}^{n-m+k}$ denotes the estimation of $\mathbf{w}_{1}$. Let $\mathbf{K}_{1}=\left[\begin{array}{c}\mathbf{0} \\ \mathbf{K}_{12} \mathbf{F}_{2}^{+}\end{array}\right]$where the gain matrix $\mathbf{K}_{12} \in \mathbb{R}^{k \times k}$ designed by the user is invertible. From

$$
\begin{aligned}
& {\left[\begin{array}{cc}
\mathbf{I}_{n-m} & \mathbf{0} \\
\mathbf{0} & \mathbf{0}
\end{array}\right]+\left[\begin{array}{c}
\mathbf{0} \\
\mathbf{K}_{12} \mathbf{F}_{2}^{+}
\end{array}\right]\left[\begin{array}{ll}
\mathbf{C}_{2} & \mathbf{F}_{2}
\end{array}\right]} \\
& \quad=\mathbf{E}_{1}+\mathbf{K}_{1} \mathbf{C}_{n}=\left[\begin{array}{cc}
\mathbf{I}_{n-m} & \mathbf{0} \\
\mathbf{K}_{12} \mathbf{F}_{2}^{+} \mathbf{C}_{2} & \mathbf{K}_{12}
\end{array}\right],
\end{aligned}
$$

we know that the matrix $\left(\mathbf{E}_{1}+\mathbf{K}_{1} \mathbf{C}_{n}\right)$ is invertible. Adding $\mathbf{K}_{1} \dot{\mathbf{y}}_{2}$ in both sides of the first equation in (22) yields

$$
\begin{aligned}
\left(\mathbf{E}_{1}+\mathbf{K}_{1} \mathbf{C}_{n}\right) \dot{\mathbf{w}}_{1}(t)= & \left(\mathbf{H}_{1}+\mathbf{L}_{1} \mathbf{C}_{n}\right) \mathbf{w}_{1}(t)+\mathbf{N}_{1} \mathbf{y}_{1}(t) \\
& +\mathbf{K}_{1} \dot{\mathbf{y}}_{2}(t)-\mathbf{L}_{1} \mathbf{y}_{2}(t)+\mathbf{M}_{1} \mathbf{n}(t)
\end{aligned}
$$

Substituting $\eta=\widehat{\mathbf{w}}_{1}-\left(\mathbf{E}_{1}+\mathbf{K}_{1} \mathbf{C}_{n}\right)^{-1} \mathbf{K}_{1} \mathbf{y}_{2}$ into (22), we can obtain

$$
\begin{aligned}
\left(\mathbf{E}_{1}+\mathbf{K}_{1} \mathbf{C}_{n}\right) \dot{\hat{\mathbf{w}}}_{1}(t)= & \left(\mathbf{H}_{1}+\mathbf{L}_{1} \mathbf{C}_{n}\right) \hat{\mathbf{w}}_{1}(t) \\
& -\mathbf{L}_{1} \mathbf{y}_{2}(t)+\mathbf{K}_{1} \dot{\mathbf{y}}_{2}(t)+\mathbf{N}_{1} \mathbf{y}_{1}(t)
\end{aligned}
$$

Let $\widetilde{\mathbf{w}}_{1}=\mathbf{w}_{1}-\widehat{\mathbf{w}}_{1}=\left[\begin{array}{c}\tilde{\mathbf{z}}_{2} \\ \widetilde{\mathbf{n}}\end{array}\right]$ be the estimation error of $\mathbf{w}_{1}$. It follows from (25) and (26) that the dynamic equation of $\tilde{\mathbf{w}}_{1}$ can be given by

$$
\begin{aligned}
\dot{\tilde{\mathbf{w}}}_{1}(t)= & \left(\mathbf{E}_{1}+\mathbf{K}_{1} \mathbf{C}_{n}\right)^{-1}\left(\left(\mathbf{H}_{1}+\mathbf{L}_{1} \mathbf{C}_{n}\right) \tilde{\mathbf{w}}_{1}(t)+\mathbf{M}_{1} \mathbf{n}(t)\right) \\
= & \left(\left(\mathbf{E}_{1}+\mathbf{K}_{1} \mathbf{C}_{n}\right)^{-1} \mathbf{H}_{1}-\mathbf{G}_{1} \mathbf{C}_{n}\right) \tilde{\mathbf{w}}_{1}(t) \\
& +\left(\mathbf{E}_{1}+\mathbf{K}_{1} \mathbf{C}_{n}\right)^{-1} \mathbf{M}_{1} \mathbf{n}(t),
\end{aligned}
$$

where $\mathbf{G}_{1}=-\left(\mathbf{E}_{1}+\mathbf{K}_{1} \mathbf{C}_{n}\right)^{-1} \mathbf{L}_{1} \in \mathbb{R}^{(n-m+k) \times(p-m)}$. By direct calculation, we have

$$
\begin{aligned}
\left(\mathbf{E}_{1}+\mathbf{K}_{1} \mathbf{C}_{n}\right)^{-1} \mathbf{M}_{1} & =\left[\begin{array}{cc}
\mathbf{I}_{n-m} & \mathbf{0} \\
-\mathbf{F}_{2}^{+} \mathbf{C}_{2} & \mathbf{K}_{12}^{-1}
\end{array}\right]\left[\begin{array}{c}
\mathbf{0} \\
\mathbf{I}_{k}
\end{array}\right]=\left[\begin{array}{c}
\mathbf{0} \\
\mathbf{K}_{12}^{-1}
\end{array}\right], \\
\left(\mathbf{E}_{1}+\mathbf{K}_{1} \mathbf{C}_{n}\right)^{-1} \mathbf{H}_{1} & =\left[\begin{array}{cc}
\mathbf{I}_{n-m} & \mathbf{0} \\
-\mathbf{F}_{2}^{+} \mathbf{C}_{2} & \mathbf{K}_{12}^{-1}
\end{array}\right]\left[\begin{array}{cc}
\mathbf{A}_{22} & \mathbf{0} \\
\mathbf{0} & -\mathbf{I}_{k}
\end{array}\right] \\
& =\left[\begin{array}{cc}
\mathbf{A}_{22} & \mathbf{0} \\
-\mathbf{F}_{2}^{+} \mathbf{C}_{2} \mathbf{A}_{22} & -\mathbf{K}_{12}^{-1}
\end{array}\right],
\end{aligned}
$$

and then rewrite (27) as

$$
\begin{aligned}
\dot{\tilde{\mathbf{w}}}_{1}(t)= & \left(\left[\begin{array}{cc}
\mathbf{A}_{22} & \mathbf{0} \\
-\mathbf{F}_{2}^{+} \mathbf{C}_{2} \mathbf{A}_{22} & -\mathbf{K}_{12}^{-1}
\end{array}\right]-\left[\begin{array}{l}
\mathbf{G}_{11} \\
\mathbf{G}_{12}
\end{array}\right]\left[\begin{array}{ll}
\mathbf{C}_{2} & \mathbf{F}_{2}
\end{array}\right]\right) \\
& \times \tilde{\mathbf{w}}_{1}(t)+\left[\begin{array}{c}
\mathbf{0} \\
\mathbf{K}_{12}^{-1}
\end{array}\right] \mathbf{n}(t) .
\end{aligned}
$$

As a result, a high-gain $\mathbf{K}_{12}$ is first chosen to reduce the effect of $\mathbf{n}$ and another gain $\mathbf{G}_{1}$ is then designed to ensure the stability of the error dynamics. The estimation performance of the developed observer is shown in the following theorem.

Theorem 2. Consider system (22), which is completely detectable, and design the PD observer as (23). Let $\hat{\mathbf{w}}_{1}=$ $\left[\begin{array}{c}\hat{\mathbf{z}}_{2} \\ \hat{\mathbf{n}}\end{array}\right]=\eta+\left(\mathbf{E}_{1}+\mathbf{K}_{1} \mathbf{C}_{n}\right)^{-1} \mathbf{K}_{1} \mathbf{y}_{2}$ be the estimation state where the dynamics of estimation error is given by (29). If the measurement noise $\mathbf{n}$ is bounded and the matrix $\left(\mathbf{E}_{1}+\mathbf{K}_{1} \mathbf{C}_{n}\right)^{-1} \mathbf{H}_{1}-\mathbf{G}_{1} \mathbf{C}_{n}$ is stable, then the estimation error is bounded within a small region. 
Proof. First, we show that the pair $\left(\mathbf{C}_{n},\left(\mathbf{E}_{1}+\mathbf{K}_{1} \mathbf{C}_{n}\right)^{-1} \mathbf{H}_{1}\right)$ is detectable. From Lemma 1 and

$$
\begin{aligned}
& \operatorname{rank}\left(\left[\begin{array}{c}
s \mathbf{I}_{n-m+k}-\left(\mathbf{E}_{1}+\mathbf{K}_{1} \mathbf{C}_{n}\right)^{-1} \mathbf{H}_{1} \\
\mathbf{C}_{n}
\end{array}\right]\right) \\
& =\operatorname{rank}\left(\left[\begin{array}{c}
s\left(\mathbf{E}_{1}+\mathbf{K}_{1} \mathbf{C}_{n}\right)-\mathbf{H}_{1} \\
\mathbf{C}_{n}
\end{array}\right]\right) \\
& =\operatorname{rank}\left(\left[\begin{array}{cc}
\mathbf{I}_{n-m+k} & s \mathbf{K}_{1} \\
\mathbf{0} & \mathbf{I}_{p-m}
\end{array}\right]\left[\begin{array}{c}
s \mathbf{E}_{1}-\mathbf{H}_{1} \\
\mathbf{C}_{n}
\end{array}\right]\right) \\
& =\operatorname{rank}\left(\left[\begin{array}{c}
s \mathbf{E}_{1}-\mathbf{H}_{1} \\
\mathbf{C}_{n}
\end{array}\right]\right),
\end{aligned}
$$

it follows that

$$
\begin{aligned}
& \operatorname{rank}\left(\left[\begin{array}{c}
\left.s \mathbf{I}_{n-m+k}-\left(\mathbf{E}_{1}+\mathbf{K}_{1} \mathbf{C}_{n}\right)^{-1} \mathbf{H}_{1}\right] \\
\mathbf{C}_{n}
\end{array}\right]\right) \\
& \quad=\operatorname{rank}\left(\left[\begin{array}{c}
s \mathbf{E}_{1}-\mathbf{H}_{1} \\
\mathbf{C}_{n}
\end{array}\right]\right) \\
& \quad=n-m+k \quad \forall s \in \mathbb{C}^{+} .
\end{aligned}
$$

Hence, the pair $\left(\mathbf{C}_{n},\left(\mathbf{E}_{1}+\mathbf{K}_{1} \mathbf{C}_{n}\right)^{-1} \mathbf{H}_{1}\right)$ is detectable and a gain matrix $\mathbf{G}_{1}$ should be found such that $\left(\mathbf{E}_{1}+\mathbf{K}_{1} \mathbf{C}_{n}\right)^{-1} \mathbf{H}_{1}-$ $\mathbf{G}_{1} \mathbf{C}_{n}$ is Hurwitz. Since $\mathbf{L}_{1}=-\left(\mathbf{E}_{1}+\mathbf{K}_{1} \mathbf{C}_{n}\right) \mathbf{G}_{1}$, we can conclude that $\left(\mathbf{E}_{1}+\mathbf{K}_{1} \mathbf{C}_{n}\right)^{-1}\left(\mathbf{H}_{1}+\mathbf{L}_{1} \mathbf{C}_{n}\right)$ can be stabilized and complete the proof.

We know from Theorem 2 that the derivative and proportional gains are used to attenuate the effect of disturbance and to ensure the robust stability of the estimation error dynamics, respectively. As a result, the estimation error is shown to be bounded within a small region.

Since the pair $\left(\mathbf{C}_{n},\left(\mathbf{E}_{1}+\mathbf{K}_{1} \mathbf{C}_{n}\right)^{-1} \mathbf{H}_{1}\right)$ is detectable, we should find the gain matrix $\mathbf{G}_{1}$ to stabilize the matrix $\left(\mathbf{E}_{1}+\mathbf{K}_{1} \mathbf{C}_{n}\right)^{-1} \mathbf{H}_{1}-\mathbf{G}_{1} \mathbf{C}_{n}$ by solving an observer Riccati equation:

$$
\begin{aligned}
\mathbf{G}_{1}= & \frac{1}{\beta} \mathbf{P}_{1} \mathbf{C}_{n}^{T}, \mathbf{P}_{1}\left(\left(\mathbf{E}_{1}+\mathbf{K}_{1} \mathbf{C}_{n}\right)^{-1} \mathbf{H}_{1}\right)^{T} \\
& +\left(\left(\mathbf{E}_{1}+\mathbf{K}_{1} \mathbf{C}_{n}\right)^{-1} \mathbf{H}_{1}\right) \mathbf{P}_{1}-\mathbf{P}_{1} \frac{\mathbf{C}_{n}^{T} \mathbf{C}_{n}}{\beta_{1}} \mathbf{P}_{1}+\mathbf{Q}_{1} \\
= & \mathbf{0}
\end{aligned}
$$

where $\beta_{1}>0$ is a positive scalar and $\mathbf{Q}_{1}>0$ is a positive definite matrix. According to the error dynamics (29), we can first design $\mathbf{K}_{1}$ to attenuate the effect of noise and then choose $\mathbf{G}_{1}$ to stabilize $\left(\mathbf{E}_{1}+\mathbf{K}_{1} \mathbf{C}_{n}\right)^{-1} \mathbf{H}_{1}-\mathbf{G}_{1} \mathbf{C}_{n}$.

For subsystem (21), we use the estimation information of $\mathbf{z}_{2}$ to design the sliding mode observer as follows:

$$
\dot{\hat{\mathbf{z}}}_{1}(t)=-\Phi_{1} \widetilde{\mathbf{z}}_{1}+\mathbf{A}_{11} \mathbf{C}_{1}^{-1} \mathbf{y}_{1}(t)+\mathbf{A}_{12} \widehat{\mathbf{z}}_{2}(t)+\mathbf{B}_{1} \mathbf{u}(t)+\mathbf{v}_{1}(t),
$$

where $\mathbf{v}_{1}=k_{1}\left(\widetilde{\mathbf{z}}_{1} /\left\|\tilde{\mathbf{z}}_{1}\right\|\right), \widetilde{\mathbf{z}}_{1}=\mathbf{C}_{1}^{-1} \mathbf{y}_{1}-\hat{\mathbf{z}}_{1}=\mathbf{z}_{1}-\hat{\mathbf{z}}_{1}, \Phi_{1} \in$ $\mathbb{R}^{m \times m}$ is a Hurwitz matrix designed by the user, and $k>0$ is a constant. The dynamic equation of estimation error $\tilde{\mathbf{z}}_{1}$ can be obtained by substituting (33) into (21):

$$
\dot{\tilde{\mathbf{z}}}_{1}(t)=\Phi_{1} \widetilde{\mathbf{z}}_{1}(t)+\mathbf{A}_{12} \widetilde{\mathbf{z}}_{2}(t)-k_{1} \frac{\tilde{\mathbf{z}}_{1}(t)}{\left\|\tilde{\mathbf{z}}_{1}(t)\right\|}+\mathbf{B}_{1} \mathbf{d}(t) .
$$

Since the vectors $\widetilde{\mathbf{z}}_{2}$ and $\mathbf{d}$ are bounded, Spurgeon et al. $[3,16,19]$ have shown that an ideal sliding motion takes place in finite time for a large enough scalar $k_{1}$. During the sliding motion $\widetilde{\mathbf{z}}_{1}(t)=\dot{\widetilde{\mathbf{z}}}_{1}(t)=\mathbf{0}$, the discontinuous term $\mathbf{v}_{1}(t)$ has to take on an average value to maintain the sliding motion. From the concept of equivalent control in sliding mode control, we know

$$
\mathbf{v}_{\text {leq }}(t)=\mathbf{A}_{12} \tilde{\mathbf{z}}_{2}(t)+\mathbf{B}_{1} \mathbf{d}(t),
$$

where $\mathbf{v}_{\text {leq }}$ represents the equivalent term required to maintain the sliding motion. Since the system is in the sliding mode, passing the term $\mathbf{v}_{1}=k_{1}\left(\tilde{\mathbf{z}}_{1} /\left\|\tilde{\mathbf{z}}_{1}\right\|\right)$ through a low-pass filter yields $\mathbf{v}_{\text {leq }}$. In practice, an ideal sliding mode does not exist; hence, the system trajectories chatter around the sliding manifold $[3,17]$. According to Haskara et al. [17], the discontinuous term $\mathbf{v}_{1}(t)$ comprises of the low-frequency equivalent control and the high-frequency switching chattering. Passing $\mathbf{v}_{1}(t)$ through a low-pass filter with a bandwidth greater than the system bandwidth but smaller than the switching frequency, one may obtain the equivalent term. The use of a low-pass filter for recovering the equivalent control signal was given by [17]. Continuous approximation of equivalent injection signal using a small positive scalar $\delta>0$ was also implemented in [19]. Since $\tilde{\mathbf{z}}_{2}$ is bounded within a known small region from Theorem 2, in the analysis of [19], we have

$$
\begin{gathered}
\hat{\mathbf{x}}(t)=\mathbf{T}_{1}^{-1} \mathbf{T}_{2}\left[\begin{array}{c}
\mathbf{C}_{1}^{-1} \mathbf{y}_{1}(t) \\
\hat{\mathbf{z}}_{2}(t)
\end{array}\right], \\
\widehat{\mathbf{d}}(t)=\mathbf{B}_{1}^{-1}\left(-k_{1} \frac{\widetilde{\mathbf{z}}_{1}(t)}{\left\|\tilde{\mathbf{z}}_{1}(t)\right\|+\delta}\right) \approx \mathbf{d}(t) .
\end{gathered}
$$

As a result, the proposed observer design generates not only a disturbance estimation $\hat{\mathbf{d}}$, but also a state estimation $\hat{\mathbf{x}}$.

Recall that most disturbance rejection controllers are of high-gain control design, which has the disadvantage of peaking phenomenon in control input. An important merit of the proposed method is that it can avoid the peaking phenomenon when applied to DRC. In high-gain control design, the magnitude of the disturbance estimate $\hat{\mathbf{d}}$ will peak at very large value during the transient time. To avoid the peaking phenomenon, an ideal control strategy is to turn on the disturbance rejection controller only after the disturbance estimation has almost been estimated. Using the estimations of state $\hat{\mathbf{x}}$ and disturbance $\hat{\mathbf{d}}$ in (36), we can design with the disturbance rejection control law as

$$
\mathbf{u}(t)=-\mathbf{K} \hat{\mathbf{x}}(t)-\left(1-\tanh \left(\frac{\left\|\mathbf{z}_{1}(t)-\widehat{\mathbf{z}}_{1}(t)\right\|}{\varepsilon_{1}}\right)\right) \hat{\mathbf{d}}(t),
$$


where $\varepsilon_{1}>0$ is a design constant and the gain matrix $\mathbf{K} \in$ $\mathbb{R}^{n \times m}$ is designed such that the matrix $\mathbf{A}-\mathbf{B K}$ is Hurwitz. We use the saturation action of the control input during a short time period to avoid the peaking phenomena.

\section{Disturbance Rejection Controller Design for Nonminimum Phase Systems}

In order to deal with nonminimum phase systems, Chen [8] used the well-known $\mathrm{H} \infty$ control design to estimate the disturbance in which the proposed design can take into account not only the robust stability but also sensitivity with respect to measurement noise. Jo et al. [7] introduced a new filter design into the traditional disturbance observer structure and adopted the $\mathrm{H} \infty$ synthesis technique. In this section, we combine SMO with the descriptor system approach in the observer design with reference to [20-23]. When the sliding motion is guaranteed, the auxiliary output can be obtained by passing the switching term in SMO through the continuous approximation. We integrate the auxiliary output and the reduced-order system dynamics to produce a new system which can be written as a descriptor system form. A $\mathrm{PD}$ observer is then designed to simultaneously estimate the system state and the unknown disturbance.

First, it follows from Section 3 that system (2) can be transformed into two subsystems; hence, system (11) can be rewritten as

$$
\begin{aligned}
& \dot{\mathbf{z}}_{1}(t)=\mathbf{A}_{11} \mathbf{z}_{1}(t)+\mathbf{A}_{12} \mathbf{z}_{2}(t)+\mathbf{B}_{1}(\mathbf{u}(t)+\mathbf{d}(t)), \\
& \dot{\mathbf{z}}_{2}(t)=\mathbf{A}_{21} \mathbf{z}_{1}(t)+\mathbf{A}_{22} \mathbf{z}_{2}(t), \\
& \mathbf{y}_{1}(t)=\mathbf{C}_{1} \mathbf{z}_{1}(t) \\
& \mathbf{y}_{2}(t)=\mathbf{C}_{2} \mathbf{z}_{2}(t)+\mathbf{F}_{2} \mathbf{n}(t) .
\end{aligned}
$$

Since the system is nonminimum phase, it follows from Lemma 1 that the pair $\left(\mathbf{A}_{22}, \mathbf{C}_{2}\right)$ is not detectable. To obtain the information of unknown disturbance, we first design the sliding mode observer as

$$
\begin{aligned}
\dot{\hat{\mathbf{z}}}_{1}(t) & =\mathbf{A}_{11} \mathbf{C}_{1}^{-1} \mathbf{y}_{1}(t)-\Phi_{2} \widetilde{\mathbf{z}}_{1}(t)+\mathbf{B}_{1} \mathbf{u}(t)+\mathbf{v}_{2}(t) \\
& =\left(\mathbf{A}_{11}-\Phi_{2}\right) \mathbf{z}_{1}(t)+\Phi_{2} \widehat{\mathbf{z}}_{1}(t)+\mathbf{B}_{1} \mathbf{u}(t)+\mathbf{v}_{2}(t),
\end{aligned}
$$

where $\mathbf{v}_{2}=k_{2}\left(\widetilde{\mathbf{z}}_{1} /\left\|\widetilde{\mathbf{z}}_{1}\right\|\right), \widetilde{\mathbf{z}}_{1}=\mathbf{z}_{1}-\widehat{\mathbf{z}}_{1}=\mathbf{C}_{1}^{-1} \mathbf{y}_{1}-\widehat{\mathbf{z}}_{1}$, and $\Phi_{2} \in \mathbb{R}^{m \times m}$ is a Hurwitz matrix designed by the user and $k_{2}>0$ is a constant. It follows from (38) and (39) that the dynamics of $\widetilde{\mathbf{z}}_{1}$ is given by

$$
\dot{\widetilde{\mathbf{z}}}_{1}(t)=\Phi_{2} \tilde{\mathbf{z}}_{1}(t)+\mathbf{A}_{12} \mathbf{z}_{2}(t)-k_{2} \frac{\tilde{\mathbf{z}}_{1}(t)}{\left\|\tilde{\mathbf{z}}_{1}(t)\right\|}+\mathbf{B}_{1} \mathbf{d}(t) .
$$

As in the analysis described in Section 4, the discontinuous term $\mathbf{v}_{2}(t)$ has to take on an average value to maintain the sliding motion, $\widetilde{\mathbf{z}}_{1}(t)=\dot{\widetilde{\mathbf{z}}}_{1}(t)=\mathbf{0}$. According to the concept of equivalent control in sliding mode control, (40) can be written as

$$
\mathbf{v}_{2 \mathrm{eq}}(t)=\mathbf{A}_{12} \mathbf{z}_{2}(t)+\mathbf{B}_{1} \mathbf{d}(t)
$$

where $\mathbf{v}_{2 \mathrm{eq}}$ represents the equivalent term required to maintain the sliding motion. As in the analysis of the above section, we use the continuous approximation to obtain

$$
\mathbf{y}_{3}(t)=k_{2} \frac{\tilde{\mathbf{z}}_{1}(t)}{\left\|\tilde{\mathbf{z}}_{1}(t)\right\|+\delta} \approx \mathbf{A}_{12} \mathbf{z}_{2}(t)+\mathbf{B}_{1} \mathbf{d}(t),
$$

Consider $y_{3}$ as the auxiliary output of the dynamics of $\mathbf{z}_{2}$ and then we have

$$
\begin{aligned}
& \dot{\mathbf{z}}_{2}(t)=\mathbf{A}_{21} \mathbf{z}_{1}(t)+\mathbf{A}_{22} \mathbf{z}_{2}(t)=\mathbf{A}_{21} \mathbf{C}_{1}^{-1} \mathbf{y}_{1}(t)+\mathbf{A}_{22} \mathbf{z}_{2}(t) \\
& \mathbf{y}_{2}(t)=\mathbf{C}_{2} \mathbf{z}_{2}(t)+\mathbf{F}_{2} \mathbf{n}(t) \\
& \mathbf{y}_{3}(t)=\mathbf{A}_{12} \mathbf{z}_{2}(t)+\mathbf{B}_{1} \mathbf{d}(t),
\end{aligned}
$$

or in a matrix form

$$
\begin{gathered}
\dot{\mathbf{z}}_{2}(t)=\mathbf{A}_{21} \mathbf{z}_{1}(t)+\mathbf{A}_{22} \mathbf{z}_{2}(t) \\
{\left[\begin{array}{l}
\mathbf{y}_{2}(t) \\
\mathbf{y}_{3}(t)
\end{array}\right]=\left[\begin{array}{ccc}
\mathbf{C}_{2} & \mathbf{0} & \mathbf{F}_{2} \\
\mathbf{A}_{12} & \mathbf{B}_{1} & \mathbf{0}
\end{array}\right]\left[\begin{array}{c}
\mathbf{z}_{2}(t) \\
\mathbf{d}(t) \\
\mathbf{n}(t)
\end{array}\right] .}
\end{gathered}
$$

In the following, we develop an observer algorithm using the descriptor system approach to simultaneously estimate the system state $\mathbf{z}_{2}$ and the unknown disturbance $\mathbf{d}$. Define the following matrices:

$$
\begin{gathered}
\mathbf{w}_{2}=\left[\begin{array}{l}
\mathbf{z}_{2} \\
\mathbf{d} \\
\mathbf{n}
\end{array}\right] \in \mathbb{R}^{n+k}, \\
\mathbf{E}_{2}=\left[\begin{array}{ccc}
\mathbf{I}_{n-m} & \mathbf{0} & \mathbf{0} \\
\mathbf{0} & \mathbf{0} & \mathbf{0} \\
\mathbf{0} & \mathbf{0} & \mathbf{0}
\end{array}\right] \in \mathbb{R}^{(n+k) \times(n+k),} \\
\mathbf{H}_{2}=\left[\begin{array}{ccc}
\mathbf{A}_{22} & \mathbf{0} & \mathbf{0} \\
\mathbf{0} & -\mathbf{I}_{m} & \mathbf{0} \\
\mathbf{0} & \mathbf{0} & -\mathbf{I}_{k}
\end{array}\right] \in \mathbb{R}^{(n+k) \times(n+k),} \\
\mathbf{N}_{2}=\left[\begin{array}{cc}
\mathbf{A}_{21} \mathbf{C}_{1}^{-1} \\
\mathbf{0} \\
\mathbf{0}
\end{array}\right] \in \mathbb{R}^{(n+k) \times m}, \\
\mathbf{M}_{2}=\left[\begin{array}{cc}
\mathbf{0} & \mathbf{0} \\
\mathbf{I}_{m} & \mathbf{0} \\
\mathbf{0} & \mathbf{I}_{k}
\end{array}\right] \in \mathbb{R}^{(n+k) \times(m+k),} \\
\mathbf{C}_{4}=\left[\begin{array}{ccc}
\mathbf{A}_{12} & \mathbf{B}_{1} & \mathbf{0} \\
\mathbf{C}_{2} & \mathbf{0} & \mathbf{F}_{2}
\end{array}\right] \in \mathbb{R}^{(n-m+k) \times(n+k),}
\end{gathered}
$$

and then a descriptor system form of system (44) can be written as

$$
\begin{gathered}
\mathbf{E}_{2} \dot{\mathbf{w}}_{2}(t)=\mathbf{H}_{2} \mathbf{w}_{2}(t)+\mathbf{N}_{2} \mathbf{y}_{1}(t)+\mathbf{M}_{2} \mathbf{f}(t), \\
\overline{\mathbf{y}}(t)=\mathbf{C}_{4} \mathbf{w}_{2}(t),
\end{gathered}
$$


where $\mathbf{f}=\left[\mathbf{d}^{T} \mathbf{n}^{T}\right]^{T} \in \mathbb{R}^{m+k}$. Before designing the observer for system (46), the following lemma demonstrates its detectability.

Lemma 3. If the pair $(\mathbf{C}, \mathbf{A})$ is detectable, then the descriptor system in (46) is completely detectable, that is,

$$
\begin{aligned}
& \text { (1) } \operatorname{rank}\left(\left[\begin{array}{c}
\mathrm{E}_{2}-\mathbf{H}_{2} \\
\mathbf{C}_{4}
\end{array}\right]\right)=n+k \quad \forall s \in \mathbb{C}^{+}, \\
& \text {(2) } \operatorname{rank}\left(\left[\begin{array}{c}
\mathrm{E}_{2} \\
\mathrm{C}_{4}
\end{array}\right]\right)=n+k .
\end{aligned}
$$

Proof. Following the aforementioned transformations, we have

$$
\begin{aligned}
& \operatorname{rank}\left(\left[\begin{array}{c}
s \mathbf{I}_{n}-\mathbf{A} \\
\mathbf{C}
\end{array}\right]\right)=\operatorname{rank}\left(\left[\begin{array}{cc}
s \mathbf{I}_{m}-\mathbf{A}_{11} & -\mathbf{A}_{12} \\
-\mathbf{A}_{21} & s \mathbf{I}_{n-m}-\mathbf{A}_{22} \\
\mathbf{C}_{1} & \mathbf{0} \\
\mathbf{0} & \mathbf{C}_{2}
\end{array}\right]\right) \\
& =\operatorname{rank}\left(\left[\begin{array}{c}
-\mathbf{A}_{12} \\
s \mathbf{I}_{n-m}-\mathbf{A}_{22} \\
\mathbf{C}_{2}
\end{array}\right]\right)+m \text {. }
\end{aligned}
$$

Since the pair $\left(\mathbf{A}_{11}, \mathbf{A}_{21}\right)$ is detectable, we have $\operatorname{rank}\left(\left[\begin{array}{c}-\mathbf{A}_{12} \\ s \mathbf{I}_{n-m}-\mathbf{A}_{22} \\ \mathbf{C}_{2}\end{array}\right]\right)=n-m$, for all $s \in \mathbb{C}^{+}$. From $\operatorname{rank}\left(\mathbf{B}_{1}\right)=m$ and $\operatorname{rank}\left(\mathbf{F}_{2}\right)=k$, we have

$$
\begin{aligned}
& \operatorname{rank}\left(\left[\begin{array}{c}
s \mathbf{E}_{2}-\mathbf{H}_{2} \\
\mathbf{C}_{4}
\end{array}\right]\right)=\operatorname{rank}\left(\left[\begin{array}{ccc}
s \mathbf{I}_{n-m}-\mathbf{A}_{22} & \mathbf{0} & \mathbf{0} \\
\mathbf{0} & \mathbf{I}_{m} & \mathbf{0} \\
\mathbf{0} & \mathbf{0} & \mathbf{I}_{k} \\
\mathbf{A}_{12} & \mathbf{B}_{1} & \mathbf{0} \\
\mathbf{C}_{2} & \mathbf{0} & \mathbf{F}_{2}
\end{array}\right]\right) \\
& =\operatorname{rank}\left(\left[\begin{array}{c}
-\mathbf{A}_{12} \\
s \mathbf{I}_{n-m}-\mathbf{A}_{22} \\
\mathbf{C}_{2}
\end{array}\right]\right)+m+k \\
& =n+k \quad \forall s \in \mathbb{C}^{+}, \\
& \operatorname{rank}\left(\left[\begin{array}{l}
\mathbf{E}_{2} \\
\mathbf{C}_{4}
\end{array}\right]\right)=\operatorname{rank}\left(\left[\begin{array}{ccc}
\mathbf{I}_{n-m} & \mathbf{0} & \mathbf{0} \\
\mathbf{0} & \mathbf{0} & \mathbf{0} \\
\mathbf{0} & \mathbf{0} & \mathbf{0} \\
\mathbf{A}_{12} & \mathbf{B}_{1} & \mathbf{0} \\
\mathbf{C}_{2} & \mathbf{0} & \mathbf{F}_{2}
\end{array}\right]\right) \\
& =n-m+m+k=n+k \text {. }
\end{aligned}
$$

Hence, system (46) is completely detectable. The proof of the lemma is finished.
Let $\mathbf{K}_{2}=\left[\begin{array}{cc}0 & \\ \mathbf{K}_{21} \mathbf{B}_{1}^{-1} & 0 \\ 0 & 0 \\ 0 & \mathbf{K}_{22} \mathbf{F}_{2}^{+}\end{array}\right] \in \mathbb{R}^{(n+k) \times(n-m+k)}$ where the gains $\mathbf{K}_{21} \in \mathbb{R}^{m \times m}$ and $\mathbf{K}_{22} \in \mathbb{R}^{k \times k}$ designed by the user are invertible. We substitute $\mathbf{K}_{2}$ into $\mathbf{E}_{2}+\mathbf{K}_{2} \mathbf{C}_{4}$ to attain

$$
\begin{aligned}
\mathbf{E}_{2}+\mathbf{K}_{2} \mathbf{C}_{4}= & {\left[\begin{array}{ccc}
\mathbf{I}_{n-m} & \mathbf{0} & \mathbf{0} \\
\mathbf{0} & \mathbf{0} & \mathbf{0} \\
\mathbf{0} & \mathbf{0} & \mathbf{0}
\end{array}\right] } \\
& +\left[\begin{array}{cc}
\mathbf{0} & \mathbf{0} \\
\mathbf{K}_{21} \mathbf{B}_{1}^{-1} & \mathbf{0} \\
\mathbf{0} & \mathbf{K}_{22} \mathbf{F}_{2}^{+}
\end{array}\right]\left[\begin{array}{ccc}
\mathbf{A}_{12} & \mathbf{B}_{1} & \mathbf{0} \\
\mathbf{C}_{2} & \mathbf{0} & \mathbf{F}_{2}
\end{array}\right]
\end{aligned}
$$

$$
=\left[\begin{array}{ccc}
\mathbf{I}_{n-m} & \mathbf{0} & \mathbf{0} \\
\mathbf{K}_{21} \mathbf{B}_{1}^{-1} \mathbf{A}_{12} & \mathbf{K}_{21} & \mathbf{0} \\
\mathbf{K}_{22} \mathbf{F}_{2}^{+} \mathbf{C}_{2} & \mathbf{0} & \mathbf{K}_{22}
\end{array}\right] \text {, }
$$

and know that the matrix $\mathrm{E}_{2}+\mathbf{K}_{2} \mathbf{C}_{4}$ is invertible. The PD observer for descriptor system (46) is designed as

$$
\begin{aligned}
\left(\mathbf{E}_{2}+\mathbf{K}_{2} \mathbf{C}_{4}\right) \dot{\eta}(t)= & \left(\mathbf{H}_{2}+\mathbf{L}_{2} \mathbf{C}_{4}\right) \eta(t) \\
& +\left(\left(\mathbf{H}_{2}+\mathbf{L}_{2} \mathbf{C}_{4}\right)\left(\mathbf{E}_{2}+\mathbf{K}_{2} \mathbf{C}_{4}\right)^{-1} \mathbf{K}_{2}-\mathbf{L}_{2}\right) \\
& \times \overline{\mathbf{y}}(t)+\mathbf{N}_{2} \mathbf{y}_{1}(t) \\
\widehat{\mathbf{w}}_{2}(t)= & \eta(t)+\left(\mathbf{E}_{2}+\mathbf{K}_{2} \mathbf{C}_{4}\right)^{-1} \mathbf{K}_{2} \overline{\mathbf{y}}(t),
\end{aligned}
$$

where $\eta \in \mathbb{R}^{n}$ and $\hat{\mathbf{w}}_{2}=\left[\begin{array}{c}\hat{\mathbf{z}}_{2} \\ \hat{\mathrm{d}} \\ \hat{\mathrm{n}}\end{array}\right] \in \mathbb{R}^{n+k}$ denotes the estimation of $\mathbf{w}_{2}$. The gain $\mathbf{L}_{2}$ is designed in the latter. Adding $\mathbf{K}_{2} \dot{\overline{\mathbf{y}}}$ in both sides of the first equation in (46) yields

$$
\begin{aligned}
\left(\mathbf{E}_{2}+\mathbf{K}_{2} \mathbf{C}_{4}\right) \dot{\mathbf{w}}(t)= & \left(\mathbf{H}_{2}+\mathbf{L}_{2} \mathbf{C}_{2}\right) \mathbf{w}(t)+\mathbf{K}_{2} \dot{\mathbf{y}}(t) \\
& -\mathbf{L}_{2} \overline{\mathbf{y}}(t)+\mathbf{N}_{2} \mathbf{y}_{1}(t)+\mathbf{M}_{2} \mathbf{f}(t),
\end{aligned}
$$

and then substituting $\eta=\widehat{\mathbf{w}}_{2}-\left(\mathbf{E}_{2}+\mathbf{K}_{2} \mathbf{C}_{4}\right)^{-1} \mathbf{K}_{2} \overline{\mathbf{y}}$ into (51) gives

$$
\begin{aligned}
\left(\mathbf{E}_{2}+\mathbf{K}_{2} \mathbf{C}_{4}\right) \dot{\hat{\mathbf{w}}}(t)= & \left(\mathbf{H}_{2}+\mathbf{L}_{2} \mathbf{C}_{4}\right) \hat{\mathbf{w}}(t)-\mathbf{L}_{2} \overline{\mathbf{y}}(t) \\
& +\mathbf{K}_{2} \dot{\overline{\mathbf{y}}}(t)+\mathbf{N}_{2} \mathbf{y}_{1}(t)
\end{aligned}
$$

Let $\tilde{\mathbf{w}}_{2}=\mathbf{w}_{2}-\hat{\mathbf{w}}_{2}$ be the estimation error of $\mathbf{w}_{2}$. It follows from (52) and (53) that the dynamics of $\widetilde{\mathbf{w}}_{2}$ is given by

$$
\begin{aligned}
\dot{\tilde{\mathbf{w}}}_{2}(t)= & \left(\mathbf{E}_{2}+\mathbf{K}_{2} \mathbf{C}_{4}\right)^{-1}\left(\left(\mathbf{H}_{2}+\mathbf{L}_{2} \mathbf{C}_{4}\right) \tilde{\mathbf{w}}_{2}(t)+\mathbf{M}_{2} \mathbf{f}(t)\right) \\
= & \left(\left(\mathbf{E}_{2}+\mathbf{K}_{2} \mathbf{C}_{4}\right)^{-1} \mathbf{H}_{2}-\mathbf{G}_{2} \mathbf{C}_{4}\right) \tilde{\mathbf{w}}_{2}(t) \\
& +\left(\mathbf{E}_{2}+\mathbf{K}_{2} \mathbf{C}_{4}\right)^{-1} \mathbf{M}_{2} \mathbf{f}(t)
\end{aligned}
$$


where $\mathbf{G}_{2}=-\left(\mathbf{E}_{2}+\mathbf{K}_{2} \mathbf{C}_{4}\right)^{-1} \mathbf{L}_{2} \in \mathbb{R}^{(n+k) \times(n-m+k)}$. Simplifying the last term of (54), we can obtain

$$
\begin{aligned}
\left(\mathbf{E}_{2}+\mathbf{K}_{2} \mathbf{C}_{4}\right)^{-1} \mathbf{M}_{2} & =\left[\begin{array}{ccc}
\mathbf{I}_{n-m} & \mathbf{0} & \mathbf{0} \\
\mathbf{B}_{1}^{-1} \mathbf{A}_{12} & \mathbf{K}_{21}^{-1} & \mathbf{0} \\
\mathbf{F}_{2}^{+} \mathbf{C}_{2} & \mathbf{0} & \mathbf{K}_{22}^{-1}
\end{array}\right]\left[\begin{array}{cc}
\mathbf{0} & \mathbf{0} \\
\mathbf{I}_{m} & \mathbf{0} \\
\mathbf{0} & \mathbf{I}_{k}
\end{array}\right] \\
& =\left[\begin{array}{cc}
\mathbf{0} & \mathbf{0} \\
\mathbf{K}_{21}^{-1} & \mathbf{0} \\
\mathbf{0} & \mathbf{K}_{22}^{-1}
\end{array}\right],
\end{aligned}
$$

and then rewrite (54) as

$$
\begin{aligned}
\dot{\tilde{\mathbf{w}}}_{2}(t)= & \left(\left(\mathbf{E}_{2}+\mathbf{K}_{2} \mathbf{C}_{4}\right)^{-1} \mathbf{H}_{2}-\mathbf{G}_{2} \mathbf{C}_{4}\right) \tilde{\mathbf{w}}_{2}(t) \\
& +\left[\begin{array}{cc}
\mathbf{0} & \mathbf{0} \\
\mathbf{K}_{21}^{-1} & \mathbf{0} \\
\mathbf{0} & \mathbf{K}_{22}^{-1}
\end{array}\right]\left[\begin{array}{l}
\mathbf{d}(t) \\
\mathbf{n}(t)
\end{array}\right] .
\end{aligned}
$$

Theorem 4. Consider system (46) where it is completely detectable and design the PD observer as (51). The dynamics of estimation error is given by (56). If the measurement noise $\mathbf{n}$ and the unknown disturbance $\mathbf{d}$ are bounded and the matrix $\left(\mathbf{E}_{2}+\mathbf{K}_{2} \mathbf{C}_{4}\right)^{-1} \mathbf{H}_{2}-\mathbf{G}_{2} \mathbf{C}_{4}$ is stable, then we first choose gains $\mathbf{K}_{21}$ and $\mathbf{K}_{22}$ to reduce the effects of $\mathbf{d}$ and $\mathbf{n}$, and then design a gain $\mathbf{G}_{2}$ to ensure the stability of the error dynamics. Hence, the estimation error is bounded within a small region.

Proof. We show that the pair $\left(\mathbf{C}_{4},\left(\mathbf{E}_{2}+\mathbf{K}_{2} \mathbf{C}_{4}\right)^{-1} \mathbf{H}_{2}\right)$ is detectable. From direct calculation, we derive the following relationship

$$
\begin{aligned}
& \operatorname{rank}\left(\left[\begin{array}{c}
s \mathbf{I}_{n+k}-\left(\mathbf{E}_{2}+\mathbf{K}_{2} \mathbf{C}_{4}\right)^{-1} \mathbf{H}_{2} \\
\mathbf{C}_{4}
\end{array}\right]\right) \\
& =\operatorname{rank}\left(\left[\begin{array}{c}
s\left(\mathbf{E}_{2}+\mathbf{K}_{2} \mathbf{C}_{4}\right)-\mathbf{H}_{2} \\
\mathbf{C}_{4}
\end{array}\right]\right) \\
& =\operatorname{rank}\left(\left[\begin{array}{cc}
\mathbf{I}_{n+k} & s \mathbf{K}_{2} \\
\mathbf{0} & \mathbf{I}_{n-m+k}
\end{array}\right]\left[\begin{array}{c}
s \mathbf{E}_{2}-\mathbf{H}_{2} \\
\mathbf{C}_{4}
\end{array}\right]\right) \\
& =\operatorname{rank}\left(\left[\begin{array}{c}
s \mathbf{E}_{2}-\mathbf{H}_{2} \\
\mathbf{C}_{4}
\end{array}\right]\right) .
\end{aligned}
$$

Since the pair $(\mathbf{C}, \mathbf{A})$ is detectable, it follows from Lemma 3 that

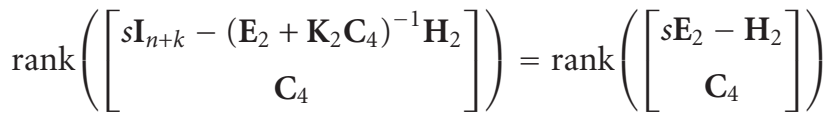

$$
\begin{aligned}
& =n+k \quad \forall s \in \mathbb{C}^{+} \text {. }
\end{aligned}
$$

As a result, the pair $\left(\mathbf{C}_{4},\left(\mathbf{E}_{2}+\mathbf{K}_{2} \mathbf{C}_{4}\right)^{-1} \mathbf{H}_{2}\right)$ is detectable and a gain matrix $\mathbf{G}_{2}$ should be found such that the matrix $\left(\mathbf{E}_{2}+\mathbf{K}_{2} \mathbf{C}_{4}\right)^{-1} \mathbf{H}_{2}-\mathbf{G}_{2} \mathbf{C}_{4}$ is Hurwitz. We can stabilize the error dynamics of $\tilde{\mathbf{w}}$ and complete the proof.
Since the pair $\left(\mathbf{C}_{4},\left(\mathbf{E}_{2}+\mathbf{K}_{2} \mathbf{C}_{4}\right)^{-1} \mathbf{H}_{2}\right)$ is detectable, similar to the work of the above section, we should find the gain matrix $\mathbf{G}_{2}$ by solving an observer Riccati equation:

$$
\begin{aligned}
\mathbf{G}_{2}= & \frac{1}{\beta_{2}} \mathbf{P}_{2} \mathbf{C}_{4}^{T}, \mathbf{P}_{2}\left(\left(\mathbf{E}_{2}+\mathbf{K}_{2} \mathbf{C}_{4}\right)^{-1} \mathbf{H}_{2}\right)^{T} \\
& +\left(\left(\mathbf{E}_{2}+\mathbf{K}_{2} \mathbf{C}_{4}\right)^{-1} \mathbf{H}_{2}\right) \mathbf{P}_{2}-\mathbf{P}_{2} \frac{\mathbf{C}_{4}^{T} \mathbf{C}_{4}}{\beta_{2}} \mathbf{P}_{2}+\mathbf{Q}_{2} \\
= & \mathbf{0},
\end{aligned}
$$

where $\beta_{2}>0$ is a positive scalar and $\mathbf{Q}_{2}>0$ is a positive definite matrix. In our proposed method, the sliding mode observer (39) is first applied to obtain the auxiliary output $\overline{\mathbf{y}}$ by passing the switching term $\mathbf{v}_{2}(t)$ through the continuous approximation (42). Then perform the descriptor system (46) comprising $\mathbf{d}, \mathbf{n}$, and the dynamics of $\mathbf{z}_{1}$. Finally, the PD observer of system (51) is employed to simultaneously estimate the system state $\mathbf{z}_{1}$ and the unknown disturbance $\mathbf{d}$. Using the estimations of state $\hat{\mathbf{x}}$ and disturbance $\hat{\mathbf{d}}$, we can finally design the disturbance rejection control law as

$$
\mathbf{u}(t)=-\mathbf{K} \widehat{\mathbf{x}}(t)-\left(1-\tanh \left(\frac{\left\|\mathbf{z}_{1}(t)-\hat{\mathbf{z}}_{1}(t)\right\|}{\varepsilon_{2}}\right)\right) \hat{\mathbf{d}}(t),
$$

where $\varepsilon_{2}>0$ is a design constant and the gain matrix $\mathbf{K} \in$ $\mathbb{R}^{n \times m}$ is designed such that the matrix $\mathbf{A}-\mathbf{B K}$ is Hurwitz.

\section{Numerical Examples}

Example 5. To demonstrate the effectiveness of the proposed method, the sensor noise and the unknown disturbance are introduced into the system and its state space form is given by

$$
\begin{aligned}
& \dot{\mathbf{x}}(t)=\left[\begin{array}{cccc}
-3.25 & -4.86 & 4.86 & 0 \\
1 & 0 & 0 & 1 \\
0 & 0.3536 & 0.3536 & -5.3571 \\
0 & 1.95 & -1.95 & 0
\end{array}\right] \mathbf{x}(t) \\
& +\left[\begin{array}{c}
2.16 \\
0 \\
0 \\
0
\end{array}\right](u(t)+\cos (t)) \\
& {\left[\begin{array}{l}
y_{1}(t) \\
y_{2}(t) \\
y_{3}(t)
\end{array}\right]=\left[\begin{array}{llll}
1 & 0 & 0 & 0 \\
0 & 1 & 0 & 0 \\
0 & 0 & 1 & 0
\end{array}\right] \mathbf{x}(t)+\left[\begin{array}{c}
0 \\
0 \\
0.5 \sin (10 t)
\end{array}\right] \text {, }}
\end{aligned}
$$


where the system satisfies three assumptions proposed in Section 2. Let $K_{12}=5, \beta_{1}=1$, and $\mathbf{Q}_{1}=\mathbf{I}$, we then obtain the PD observer as

$$
\begin{aligned}
& \dot{\eta}(t)=\left[\begin{array}{cccc}
-7.5875 & 2.0188 & 1 & 2.0188 \\
49.5806 & -16.2230 & -5.3571 & -16.5766 \\
-28.3729 & 7.8498 & 0 & 9.7998 \\
-47.5618 & 14.2794 & 5.3571 & 14.4330
\end{array}\right] \eta(t) \\
& +\left[\begin{array}{cc}
7.5875 & 0 \\
-49.2270 & 0 \\
30.3229 & 0 \\
47.2082 & -0.2
\end{array}\right]\left[\begin{array}{l}
y_{2}(t) \\
y_{3}(t)
\end{array}\right]+\left[\begin{array}{llll}
1 & 0 & 0 & 0
\end{array}\right]^{T} y_{1}(t),
\end{aligned}
$$

and the estimation is constructed as

$$
\left[\begin{array}{llll}
\hat{x}_{2}(t) & \hat{x}_{3}(t) & \hat{x}_{4}(t) & \hat{n}(t)
\end{array}\right]^{T}=\eta(t)+\left[\begin{array}{llll}
0 & 0 & 0 & 0 \\
0 & 0 & 0 & 1
\end{array}\right]^{T}\left[\begin{array}{l}
y_{2}(t) \\
y_{3}(t)
\end{array}\right] .
$$

Finally, the sliding mode observer and the disturbance rejection controller are designed as

$$
\begin{aligned}
& \dot{\hat{y}}_{1}(t)=-1 \tilde{y}_{1}(t)-3.25 y_{1}(t)+\left[\begin{array}{lll}
-4.86 & 4.86 & 0
\end{array}\right]\left[\begin{array}{l}
\hat{x}_{2}(t) \\
\hat{x}_{3}(t) \\
\hat{x}_{4}(t)
\end{array}\right] \\
& +2.16 u(t)+30 \operatorname{sign}\left(\tilde{y}_{1}(t)\right), \\
& u(t)=-\left[\begin{array}{llll}
3.7446 & 26.2338 & -35.2773 & 62.8449
\end{array}\right]\left[\begin{array}{l}
y_{1}(t) \\
\hat{x}_{2}(t) \\
\hat{x}_{3}(t) \\
\hat{x}_{4}(t)
\end{array}\right] \\
& -\frac{1}{2.16}\left(1-\tanh \left(\frac{\tilde{y}_{1}(t)}{0.05}\right)\right) \frac{30 \tilde{y}_{1}(t)}{\left|\tilde{y}_{1}(t)\right|+0.01} .
\end{aligned}
$$

Since the states $x_{1}, x_{2}$, and $x_{3}$ are available, Figure 1 illustrates the responses of the estimation state and the true state of $x_{4}$

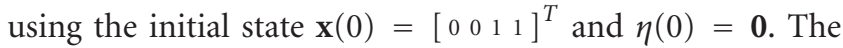
true and estimation of disturbance are shown in Figure 2. Although the system has the sensor noise, our method can obtain better estimation response as seen in these figures. Figures 3 and 4 are the responses of the system states and the control input, respectively. Our proposed disturbance rejection controller does not have the drawback of peaking, and the disturbance attenuation property is evident. Hence, the proposed observer is capable of estimating the state when the underlying system has both unknown disturbance and measurement noise.

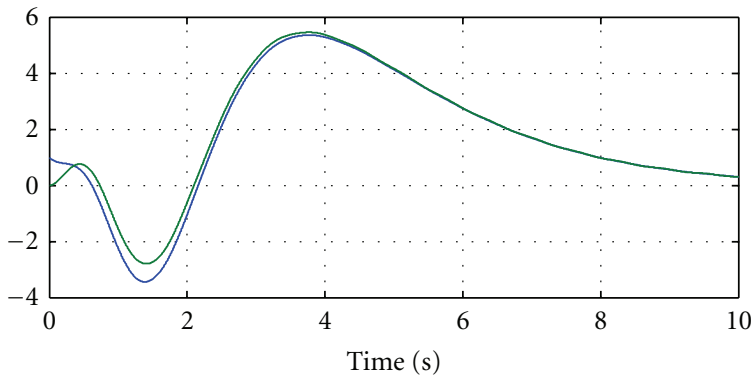

$$
\text { Estimation of } x_{4}
$$

Figure 1: True and estimation values of $x_{4}$ for Example 5.

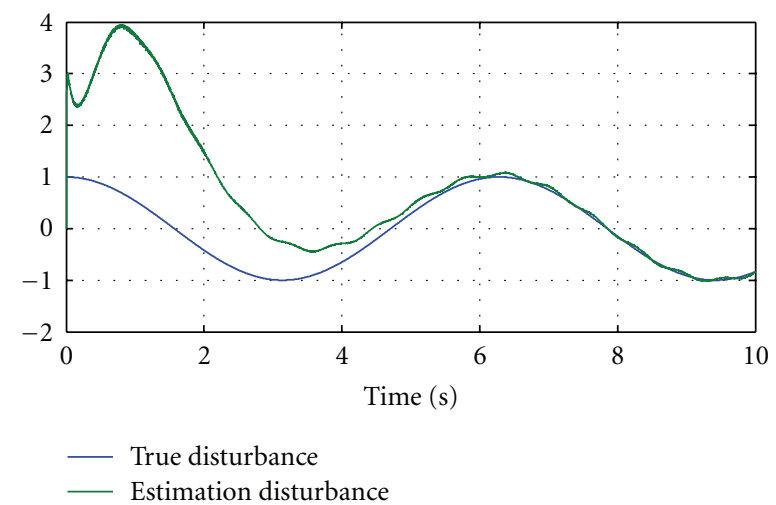

Figure 2: True and estimation values of disturbance for Example 5.

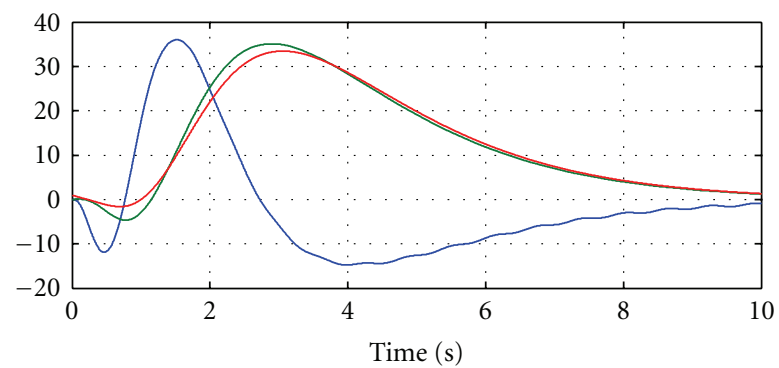

$x_{1}$
$-x_{2}$
$-x_{3}$

Figure 3: Responses of system states $x_{1}, x_{2}$, and $x_{3}$ for Example 5.

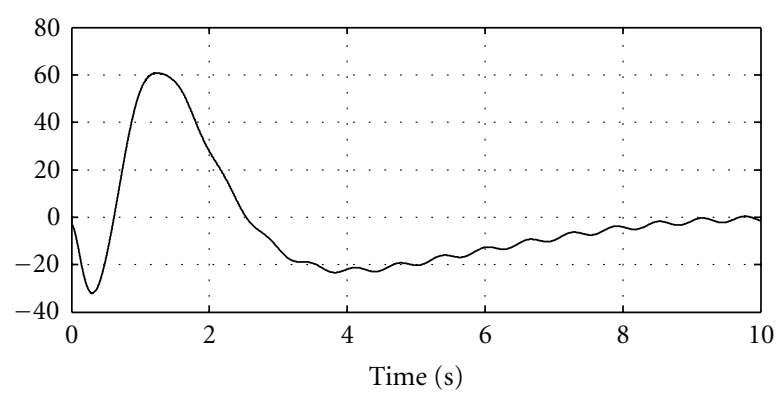

Figure 4: Control input for Example 5. 


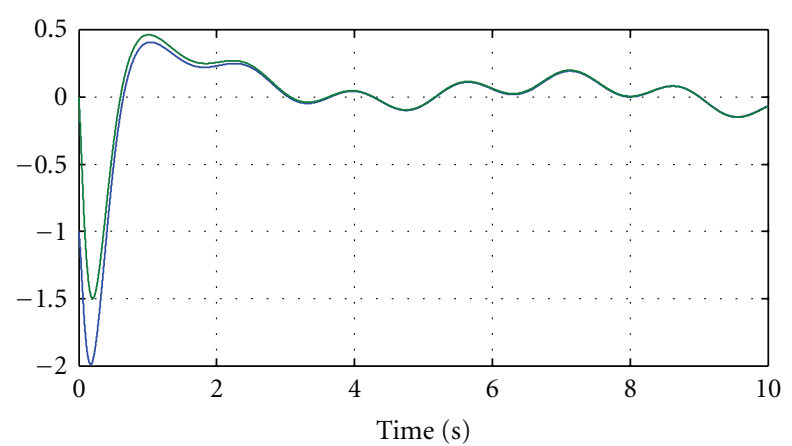

$-x_{1}$

— Estimation of $x_{1}$

Figure 5: True and estimation values of $x_{1}$ for Example 6.

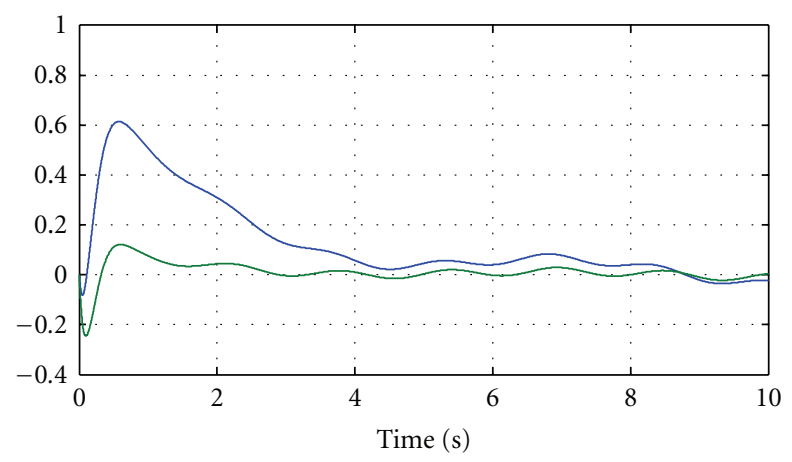

- Estimation of $x_{2}$

Figure 6: True and estimation values of $x_{2}$ for Example 6.

Example 6. To demonstrate the proposed observer design method in Section 5, we consider the following system:

$$
\begin{aligned}
\dot{\mathbf{x}}(t)= & {\left[\begin{array}{cccc}
-4 & -0.4 & -3.2 & -5.3 \\
-2.4 & -0.2 & -6.4 & 0.3470 \\
1 & -10 & -0.25 & -1.04 \\
1 & -8 & 3 & -0.96
\end{array}\right] \mathbf{x}(t) } \\
& +\left[\begin{array}{cc}
0 & 0 \\
0 & 0 \\
-1.2 & -0.4 \\
4 & 9.3
\end{array}\right]\left(\mathbf{u}(t)+\left[\begin{array}{c}
\sin (4 t) \\
\cos (t)
\end{array}\right]\right), \\
\mathbf{y}(t)= & {\left[\begin{array}{llll}
0 & 0 & 1 & 0 \\
0 & 0 & 0 & 1
\end{array}\right] \mathbf{x}(t) . }
\end{aligned}
$$

By direct calculation, we know that the eigenvalues of the system are $\{-9.3421,-2.9878,3.46 \pm 0.9983 i\}$ and the invariant zeros of the system between the output and the unknown disturbance are $\{0.0378,-4.2378\}$. As a result, the system is unstable and has unstable invariant zero. The conventional UIO [10-13] and SMO [15-17] methods

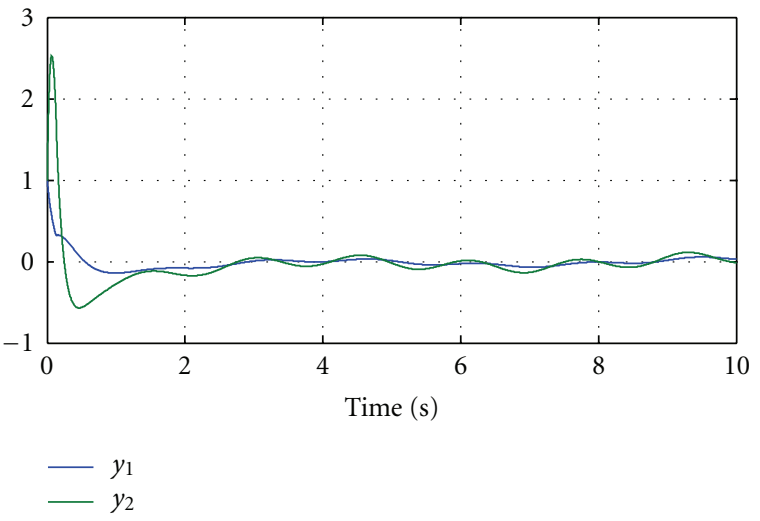

Figure 7: System outputs $y_{1}$ and $y_{2}$ for Example 6.

cannot be directly implemented in this system. Following the design procedures in Section 5, the sliding mode observer and the continuous approximation are designed as

$$
\begin{aligned}
\dot{\hat{\mathbf{y}}}(t)= & 3(\mathbf{y}(t)-\hat{\mathbf{y}}(t))+\left[\begin{array}{cc}
-0.25 & -1.04 \\
3 & -0.96
\end{array}\right] \mathbf{y}(t) \\
& +15 \frac{(\mathbf{y}(t)-\hat{\mathbf{y}}(t))}{\|\mathbf{y}(t)-\hat{\mathbf{y}}(t)\|}+\left[\begin{array}{cc}
-1.2 & -0.4 \\
4 & 9.3
\end{array}\right] \mathbf{u}(t) \\
\overline{\mathbf{y}}(t)= & 15 \frac{(\mathbf{y}(t)-\hat{\mathbf{y}}(t))}{\|\mathbf{y}(t)-\hat{\mathbf{y}}(t)\|+0.01} .
\end{aligned}
$$

Designing $\mathbf{F}_{2}=2, \beta=1$, and $\mathbf{Q}=\mathbf{I}_{4}$, and solving an observer Riccati equation as in the Kalman filter, we can obtain the gain matrix $\boldsymbol{G}$. The PD observer can be given by

$$
\begin{aligned}
\dot{\eta}(t)= & {\left[\begin{array}{cccc}
-4.1803 & 1.2661 & -0.1393 & -0.5914 \\
-0.9390 & -13.8822 & 0.6581 & 3.9135 \\
18.5277 & 15.5645 & -3.0060 & -7.5507 \\
-10.0052 & -4.5997 & -3.1074 & -5.7984
\end{array}\right] \eta(t) } \\
& +\left[\begin{array}{cc}
0 & 0 \\
0 & 0 \\
0.4864 & 0.0209 \\
-0.2092 & -0.0628
\end{array}\right] \overline{\mathbf{y}}(t) \\
& +\left[\begin{array}{cc}
-3.2 & -5.3 \\
-6.4 & 0.3470 \\
61.1548 & -8.8694 \\
-31.4644 & 4.6832
\end{array}\right] \mathbf{y}(t) .
\end{aligned}
$$




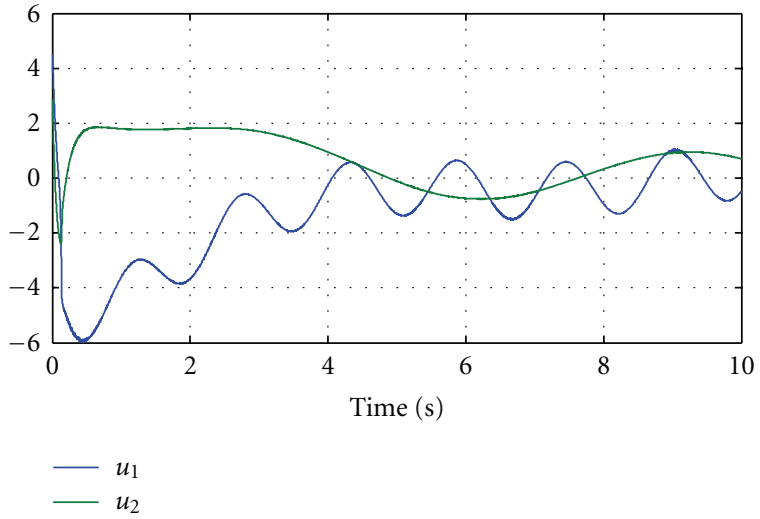

FIGURE 8: System inputs $u_{1}$ and $u_{2}$ for Example 6.

Since $x_{3}=y_{1}$ and $x_{4}=y_{2}$, we can obtain the estimations of reduced state and disturbance as

$$
\begin{aligned}
& {\left[\begin{array}{llll}
\hat{x}_{1}(t) & \hat{x}_{2}(t) & \hat{d}_{1}(t) & \hat{d}_{2}(t)
\end{array}\right]^{T}} \\
& =\eta(t)+\left[\begin{array}{rrrr}
0 & 0 & -0.9728 & 0.4184 \\
0 & 0 & -0.0418 & 0.1255
\end{array}\right]^{T} \overline{\mathbf{y}}(t) .
\end{aligned}
$$

Finally, the disturbance rejection controller is designed as

$$
\begin{aligned}
\mathbf{u}(t)= & -\left[\begin{array}{llll}
-1.7106 & 7.1062 & -5.4759 & 0.9655 \\
-1.6695 & 5.7243 & -4.3594 & 1.4846
\end{array}\right]\left[\begin{array}{l}
\hat{x}_{1}(t) \\
\hat{x}_{2}(t) \\
y_{1}(t) \\
y_{2}(t)
\end{array}\right] \\
& -\left(1-\tanh \left(\frac{\|\mathbf{y}(t)-\hat{\mathbf{y}}(t)\|}{0.1}\right)\right)\left[\begin{array}{l}
\hat{d}_{1}(t) \\
\hat{d}_{2}(t)
\end{array}\right] .
\end{aligned}
$$

Setting the initial conditions as $\mathbf{x}(0)=\left[\begin{array}{lll}-1 & 0 & 1\end{array}\right]^{T}, \widehat{\mathbf{y}}(0)=\mathbf{0}$ and $\eta(0)=\mathbf{0}$, Figures 5 and 6 are the true and estimation states. The system responses including the output and the input are shown in Figures 7 and 8. Figures 9 and 10 are the estimation and real unknown disturbances of $d_{1}$ and $d_{2}$, respectively, in which each estimated signal has approximately traced the real one. The observer design respecting to the proposed method robustly stabilize the estimation error dynamics and simultaneously reconstruct the state and unknown input even if the system is unstable and has unstable invariant zeros. Unlike the conventional high-gain disturbance rejection controllers, the control input in our proposed method does not have the disadvantage of peaking at the transient time. Moreover, the property of disturbance attenuation in our algorithm is evident.

\section{Conclusions}

For a class of MIMO systems with the unknown disturbances and the measurement noises, this paper has developed an

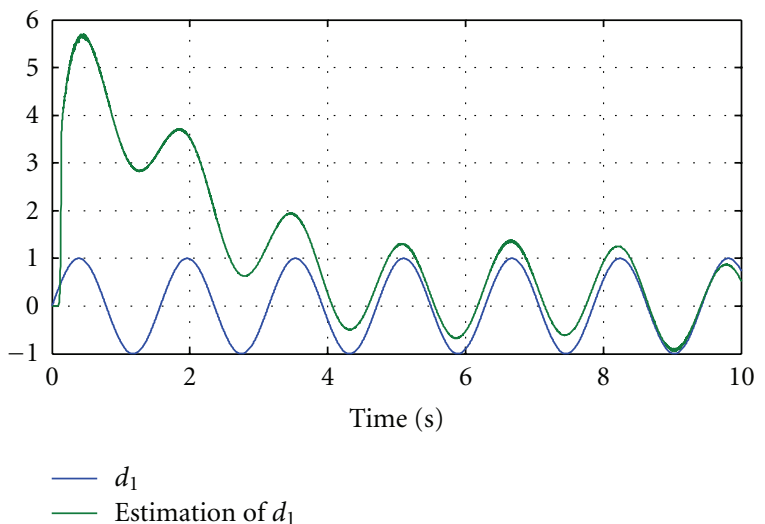

Figure 9: True and estimation values of $d_{1}$ for Example 6.

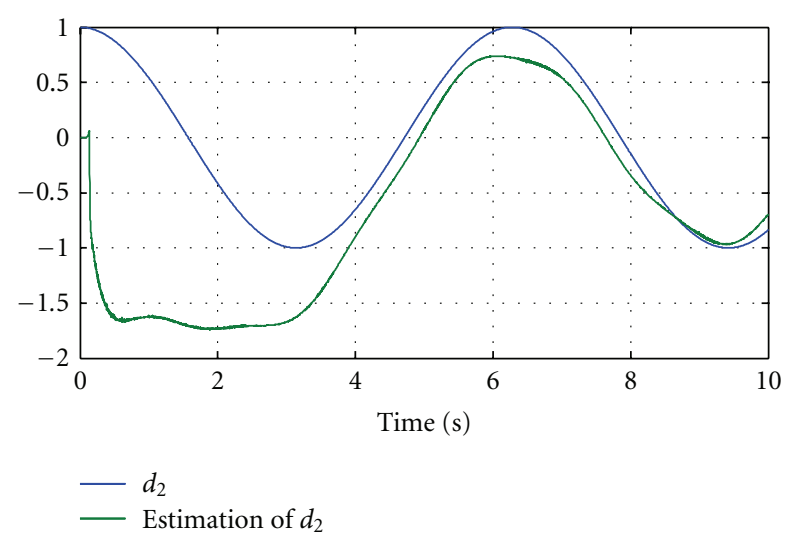

FIgURE 10: True and estimation values of $d_{2}$ for Example 6.

observer design method consisting of the sliding mode observer and the descriptor system transformation. The proposed estimation method can simultaneously reconstruct the system state and the unknown disturbance even though the system is nonminimum phase with respect to the relation between the output and the unknown disturbance. The robust stability of the estimation dynamics can be guaranteed and the estimation error is shown to be bounded within a small region. Compared with conventional highgain disturbance rejection controllers, our controller can avoid the peaking phenomenon and does not require the derivative of output. Simulation results demonstrate that the proposed control scheme exhibits reasonably good system performance.

\section{Acknowledgment}

The author acknowledges the financial support provided by the National Science Council of Taiwan, Taiwan under Grant NSC 99-2221-E-161-007.

\section{References}

[1] J. C. Willems, "Almost invariant subspaces: an approach to high gain feedback design-part I: almost controlled invariant 
subspaces," IEEE Transactions on Automatic Control, vol. 26, no. 1, pp. 235-252, 1981.

[2] K. D. Young, "Disturbance decoupling by high gain feedback," IEEE Transactions on Automatic Control, vol. 27, pp. 970-971, 1982.

[3] C. Edwards and S. K. Spurgeon, Sliding Mode Control: Theory and Application, Taylor and Francis, New York, NY, USA, 1998.

[4] Z. Lin and A. Saberi, "Robust semiglobal stabilization of minimum-phase input-output linearizable systems via partial state and output feedback," IEEE Transactions on Automatic Control, vol. 40, no. 6, pp. 1029-1041, 1995.

[5] H. J. Sussmann and P. V. Kokotovic, "The peaking phenomenon and the global stabilization of nonlinear systems," IEEE Transactions on Automatic Control, vol. 36, no. 4, pp. 424-440, 1991.

[6] O. Kouhei, M. Shibata, and T. Murakami, "Motion control for advanced mechatronics," IEEE/ASME Transactions on Mechatronics, vol. 1, no. 1, pp. 56-67, 1996.

[7] N. H. Jo, H. Shim, and Y. I. Son, "Disturbance observer for non-minimum phase linear systems," International Journal of Control, Automation and Systems, vol. 8, pp. 994-1002, 2010.

[8] M. S. Chen, Ho Disturbance observer design, Ph.D. thesis, Department of Mechanical Engineering, National Taiwan University, Taiwan, 2009.

[9] M. Corless and J. Tu, "State and input estimation for a class of uncertain systems," Automatica, vol. 34, no. 6, pp. 757-764, 1998.

[10] J. H. She, M. Fang, Y. Ohyama, H. Hashimoto, and M. Wu, "Improving disturbance-rejection performance based on an equivalent-input-disturbance approach," IEEE Transactions on Industrial Electronics, vol. 55, no. 1, pp. 380-389, 2008.

[11] C. S. Liu and H. Peng, "Inverse-dynamics based state and disturbance observers for linear time-invariant systems," Journal of Dynamic Systems, Measurement and Control, vol. 124, no. 3, pp. 375-381, 2002.

[12] M. Darouach, M. Zasadzinski, and S. J. Xu, "Full-order observers for linear systems with unknown inputs," IEEE Transactions on Automatic Control, vol. 39, no. 3, pp. 606-609, 1994.

[13] M. Hou and P. C. Muller, "Design of observers for linear systems with unknown inputs," IEEE Transactions on Automatic Control, vol. 37, no. 6, pp. 871-875, 1992.

[14] S. Mondal, G. Chakraborty, and K. Bhattacharyya, "LMI approach to robust unknown input observer design for continuous systems with noise and uncertainties," International Journal of Control, Automation and Systems, vol. 8, no. 2, pp. 210-219, 2010.

[15] R. Raoufi, Nonlinear robust observers for simultaneous state and fault estimation, Ph.D. thesis, Department of Electrical Engineering, University of Alberta, Alberta, Canada, 2010.

[16] S. K. Spurgeon, "Sliding mode observers: a survey," International Journal of Systems Science, vol. 39, no. 8, pp. 751-764, 2008.

[17] I. Haskara, U. Ozguner, and V. I. Utkin, "On sliding mode observers via equivalent control approach," International Journal of Control, vol. 71, no. 6, pp. 1051-1067, 1998.

[18] K. Y. Ng, C. P. Tan, Z. Man, and R. Akmeliawati, "New results in disturbance decoupled fault reconstruction in linear uncertain systems using two sliding mode observers in cascade," International Journal of Control, Automation and Systems, vol. 8, no. 3, pp. 506-518, 2010.

[19] C. Edwards, S. K. Spurgeon, and R. J. Patton, "Sliding mode observers for fault detection and isolation," Automatica, vol. 36, no. 4, pp. 541-553, 2000.
[20] M. Boutayeb, M. Darouach, and H. Rafaralahy, "Generalized state-space observers for chaotic synchronization and secure communication," IEEE Transactions on Circuits and Systems I, vol. 49, no. 3, pp. 345-349, 2002.

[21] T. Fernando and H. Trinh, "Design of reduced-order state/unknown input observers based on a descriptor system approach," Asian Journal of Control, vol. 9, no. 4, pp. 458-465, 2007.

[22] Z. Gao and H. Wang, "Descriptor observer approaches for multivariable systems with measurement noises and application in fault detection and diagnosis," Systems and Control Letters, vol. 55, no. 4, pp. 304-313, 2006.

[23] A. G. Wu and G. R. Duan, "Design of PD observers in descriptor linear systems," International Journal of Control, Automation and Systems, vol. 5, no. 1, pp. 93-98, 2007.

[24] K. K. Busawon and P. Kabore, "Disturbance attenuation using proportional integral observers," International Journal of Control, vol. 74, no. 6, pp. 618-627, 2001.

[25] M. Aldeen and R. Sharma, "Estimation of states, faults and unknown disturbances in non-linear systems," International Journal of Control, vol. 81, no. 8, pp. 1195-1201, 2008. 

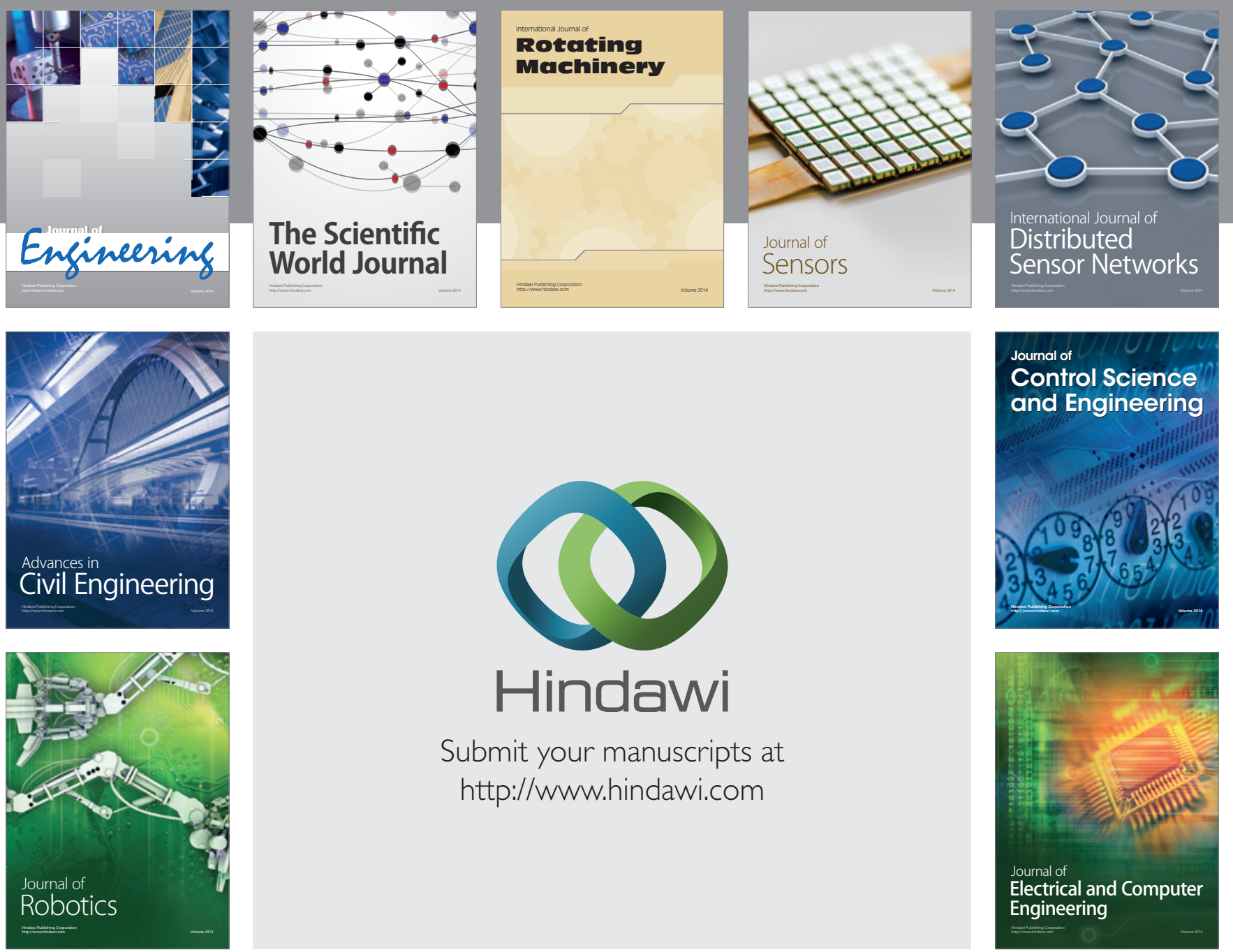

Submit your manuscripts at

http://www.hindawi.com
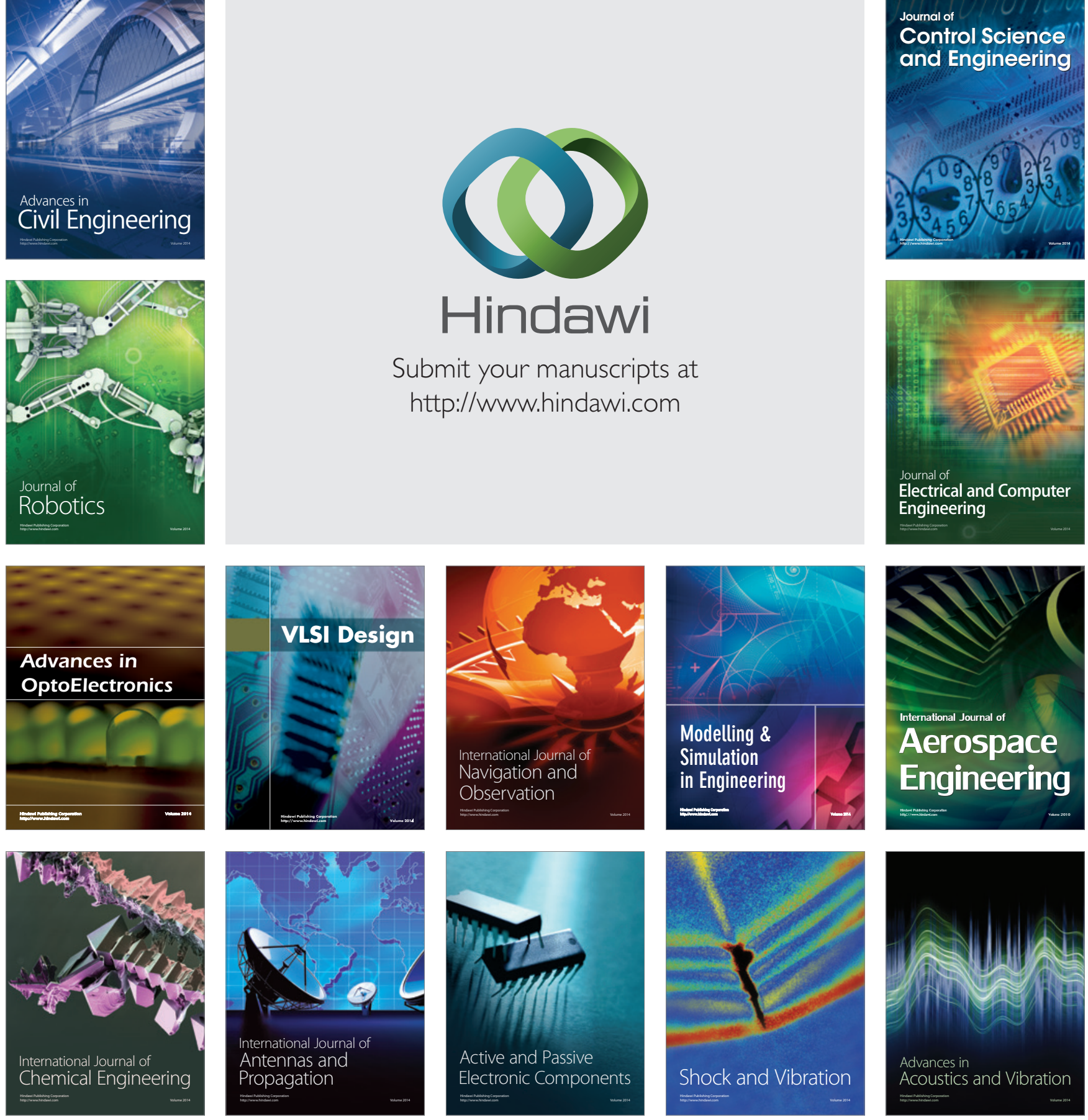